Statistical Methods in Medical Research

\title{
A Bayesian approach for the identification of patient-specific parameters in a dialysis kinetic model
}

\author{
Camilla Bianchi, ' Ettore Lanzarone, ${ }^{2}$ Giustina Casagrande' and \\ Maria Laura Costantino'
}

\begin{abstract}
Hemodialysis is the most common therapy to treat renal insufficiency. However, notwithstanding the recent improvements, hemodialysis is still associated with a non-negligible rate of comorbidities, which could be reduced by customizing the treatment. Many differential compartment models have been developed to describe the mass balance of blood electrolytes and catabolites during hemodialysis, with the goal of improving and controlling hemodialysis sessions. However, these models often refer to an average uremic patient, while on the contrary the clinical need for customization requires patient-specific models. In this work, we assume that the customization can be obtained by means of patient-specific model parameters. We propose and validate a Bayesian approach to estimate the patientspecific parameters of a multi-compartment model, and to predict the single patient's response to the treatment, in order to prevent intra-dialysis complications. The likelihood function is obtained by means of a discretized version of the multi-compartment model, where the discretization is in terms of a Runge-Kutta method to guarantee convergence, and the posterior densities of model parameters are obtained through Markov Chain Monte Carlo simulation. Results show fair estimations and the applicability in the clinical practice.
\end{abstract}

\section{Keywords}

Hemodialysis, patient-specific response, Bayesian estimation approach

\section{Introduction}

Hemodialysis (HD) is the elective option to treat End Stage Renal Disease (ESRD). However, despite the improvements made in the last years, HD is still associated with a non-negligible rate of comorbidities. ${ }^{1,2}$ In particular, due to therapy discontinuity, HD induces considerable changes in osmotic balances and rapid variations in fluid volumes ${ }^{3-5}$ and electrolytic concentrations ${ }^{6}$ within patients' body compartments. Thus, HD treatments should be optimized to reduce these alterations. Moreover, a need for treatment customization emerged over the years, to reduce the associated comorbidities, because the individual tolerance to HD may vary from patient to patient also in the presence of similar treatment conditions. ${ }^{7,8}$

Instruments that simulate and predict the patient-specific response to HD treatment in terms of electrolyte and catabolite kinetics are a necessary step toward customization, in order to identify the most suitable therapy for reducing intra-dialysis complications and associated long-term dysfunctions.

In this work, we refer to the parametric multi-compartment kinetic model of Casagrande et al., ${ }^{9}$ and we assume that the customization can be obtained by means of patient-specific model parameters, which modulate the mass and fluid balance across the main membranes involved in HD process. We propose and validate a Bayesian estimation approach to determine the patient-specific parameters of this multi-compartment model and to predict the single patient's response to HD treatment. The likelihood function is obtained by means of a

\footnotetext{
'Department of Chemistry, Materials and Chemical Engineering, Politecnico di Milano, Milan, Italy

${ }^{2}$ Istituto di Matematica Applicata e Tecnologie Informatiche (IMATI), Consiglio Nazionale delle Ricerche (CNR), Milan, Italy

Corresponding author:

Camilla Bianchi, Laboratory of Biological Structure Mechanics, Chemistry, Materials and Chemical Engineering Department, Politecnico di Milano, P.za Leonardo da Vinci 32, 20133 Milano, Italy.

Email: camilla.bianchi@polimi.it
} 
discretized version of the above mentioned multi-compartment model, in which the discretization is performed in terms of a Runge-Kutta method to guarantee the convergence, while the posterior densities of model parameters are obtained through Markov Chain Monte Carlo (MCMC) simulation.

Differently from Casagrande et al., ${ }^{9}$ where a constrained non-linear optimization algorithm was used to get the parameter values, the Bayesian approach allows us to formally include the clinical prior knowledge on the parameters and to directly evaluate the uncertainty associated with the estimates, by means of their posterior probability density functions.

The paper is structured as follows. We first present a literature analysis on both HD kinetic models and Bayesian estimation approaches in dynamic systems. Then, we briefly describe the adopted kinetic model ${ }^{9}$ and we detail the Bayesian approach to estimate the patient-specific model parameters. Afterwards, we show the computational validation of the approach and the results from the application to real patients. We conclude the paper discussing the application of the optimized model (with patient-specific parameters) in the clinical practice.

Data used for both the validation and the application to real patients have been obtained within the DialysIS Project.

\section{Literature analysis}

In this section, we first revise the literature dealing with mathematical compartment models to describe the patient's kinetics during the HD treatment. Then, we overview some works including Bayesian approaches to estimate the parameters in dynamic models.

\section{I Dialysis modeling}

Several mathematical models have been proposed in the literature to describe solute kinetics during HD and to assist clinicians in individualizing HD prescriptions. For these models, it has been proved that, to properly model mass and fluid exchanges during HD, it is highly important to consider solutes compartmentalization. ${ }^{10}$ First, the single-pool model had been applied to simulate the kinetics of small molecules (mainly urea), ${ }^{11-14}$ providing a method for efficiently quantifying the target hemodialysis dose. Then, it became clear that two- or multiple-pool models better simulate the kinetics of small solutes not freely permeable, e.g. creatinine and phosphate, and the kinetics of larger molecules, e.g. $\beta 2$-microglobulin. ${ }^{15}$ Several tests on single-pool variable volume or pseudo-one compartment models have been performed for the assessment of intradialytic mass balance of single solutes, e.g. calcium, potassium and phosphorus, that play a crucial role for hydro-electrolyte equilibria. ${ }^{16-18}$ To achieve more effective prediction models, multi-pool models have been introduced, allowing to describe the simultaneous contribution of different plasma solutes. ${ }^{19}$ However, none of these models aim at characterizing the patientspecific solute kinetics in HD, apart from Ursino et al., ${ }^{19}$ in which an individual estimation of few parameters characterizing solute kinetics has been performed.

On the contrary, the clinical need for customization requires patient-specific models. In particular, as done in this work, the customization can be obtained by including patient-specific parameters in models that are common to all patients. This respects the idea that the physio-pathological mechanisms are the same, while each patient is characterized by different parameters that tune the behavior (e.g. biorheological parameters and peripheral resistances). Moreover, this assumption allows to exploit the existing models as clinical predictors while pursuing HD personalization.

The only available work that includes a patient-specific characterization of solute kinetics in HD is that of Casagrande et al., ${ }^{9}$ where the estimates have been obtained by minimizing a weighted least-square criterion function of the differences between clinical data and model predictions. However, in the context of HD, this approach shows two limitations. First, estimates are given in terms of a punctual value, which does not allow to assess if two different estimates are significantly different or not. Second, the least-square minimization does not allow to consider any prior information different from a sub-domain in the parameter space. In the clinical context, where the patient-specific customization relies on both literature data and few observations from the patient, the possibility of including a prior belief (as in the Bayesian framework) is an added value.

\subsection{Bayesian approaches for parameter estimation}

The temporal evolution of continuous dynamic systems is usually modeled by means of differential equations. When randomness affects the system evolution, we refer to stochastic differential equations (SDEs) and stochastic 


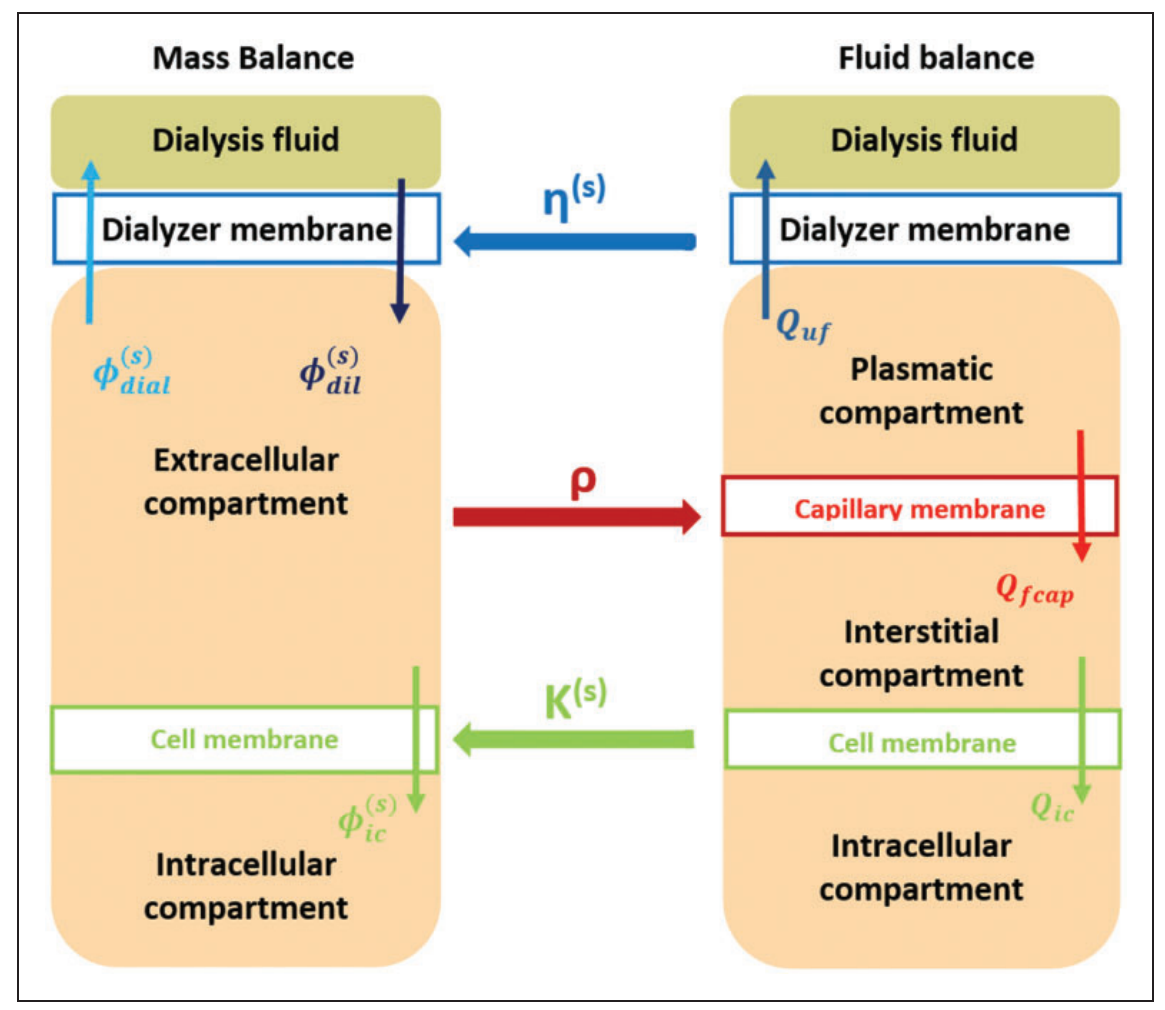

Figure I. Body compartments of a HD patient with fluid and mass exchanges across the biological and artificial membranes (patientspecific parameters $\rho, k^{(s)}$ and $\eta^{(s)}$ are highlighted where they act).

partial differential equations (SPDEs). Randomness can be considered in different parts of the equation, e.g. parameters, forcing terms, initial conditions, and boundary conditions.

SDEs and SPDEs started almost 50 years ago and are becoming more and more popular for the description of random phenomena in different applicative fields, from physics to engineering and biology. Theoretical aspects of SDEs and SPDEs are largely described in Oksendal, ${ }^{20}$ while numerical issues are discussed in Kloeden and Platen. ${ }^{21}$ Their use for reverse engineering problems, in which observations and/or measurements are converted into knowledge of the structure and the parameters of the system, is largely documented.

In particular, Bayesian approaches have been successfully applied to estimate the parameters (in terms of their stochastic probability density function) and the stochastic response of SDEs and SPDEs in different fields. ${ }^{22}$ Indeed, once the stochastic characterization of model parameters has been conducted, SDEs and SPDEs can be solved, and the result is another stochastic process. ${ }^{20,21}$

For example, they have been applied to estimate the inertance in a hydraulic simulator of the human circulation, ${ }^{23}$ the parameters in a stochastic predator-prey system, ${ }^{24}$ the thermal conductivity and the temperature profile in polymers, ${ }^{25}$ and the aortic stiffness from non-invasive measurements. ${ }^{26}$ More recently, Bayesian approaches have been considered for estimating the parameters that characterize the dynamics of aquatic communities, ${ }^{27}$ and for the estimation of the mortality terms in a stage-structured demographic model. ${ }^{28}$ However, to the best of our knowledge, they have been never applied to characterize the dynamics of plasmatic electrolytes and, more in general, they have never been applied to HD patients.

\section{Multi-compartment kinetic model}

We consider the multi-compartment kinetic model of Casagrande et al. ${ }^{9}$ and assume that the customization is given in terms of patient-specific model parameters, while the structure of the model remains the same for all HD patients. An overview of the model is provided below; further details can be found in Casagrande et al. ${ }^{9}$

This model allows to evaluate, for the entire duration of a HD treatment, the temporal trend of the blood concentration for different plasmatic electrolytes and breakdown products (defined as solutes in the following), together with the temporal trend of blood volume. These are in fact the critical variables that are most related to 
Table I. Marginal posterior densities of model parameters vs. their true values (HDF-pre, HFO case).

\begin{tabular}{|c|c|c|c|c|c|c|}
\hline HDF-pre & Min & $25 \%$ & $50 \%$ & $75 \%$ & Max & true \\
\hline$\eta^{(\mathrm{I})}-$ sodium & 0.50 & 0.50 & 0.50 & 0.50 & 0.50 & 0.50 \\
\hline$\eta^{(2)}-$ potassium & 0.50 & 0.50 & 0.50 & 0.51 & 0.52 & 0.50 \\
\hline$\eta^{(3)}-$ chloride & 0.50 & 0.50 & 0.50 & 0.50 & 0.50 & 0.50 \\
\hline$\eta^{(4)}-$ calcium & 0.50 & 0.50 & 0.50 & 0.50 & 0.50 & 0.50 \\
\hline$\eta^{(5)}-$ bicarbonate & 0.44 & 0.44 & 0.44 & 0.44 & 0.45 & 0.50 \\
\hline$\eta^{(6)}-$ magnesium & 0.49 & 0.50 & 0.50 & 0.51 & 0.51 & 0.50 \\
\hline$\eta^{(7)}-$ urea & 0.47 & 0.48 & 0.48 & 0.48 & 0.49 & 0.50 \\
\hline$\eta^{(8)}-$ creatinine & 0.46 & 0.47 & 0.47 & 0.48 & 0.49 & 0.50 \\
\hline$\rho$ & 0.86 & 0.86 & 0.86 & 0.87 & 0.87 & 1.00 \\
\hline$k^{(1)}-$ sodium & $2.04 \cdot 10^{-3}$ & $2.40 \cdot 10^{-3}$ & $2.49 \cdot 10^{-3}$ & $2.60 \cdot 10^{-3}$ & $2.99 \cdot 10^{-3}$ & $2.50 \cdot 10^{-3}$ \\
\hline$k^{(2)}-$ potassium & $1.63 \cdot 10^{-4}$ & $1.68 \cdot 10^{-4}$ & $1.69 \cdot 10^{-4}$ & $1.71 \cdot 10^{-4}$ & $1.77 \cdot 10^{-4}$ & $1.67 \cdot 10^{-4}$ \\
\hline$k^{(3)}-$ chloride & $9.77 \cdot 10^{-5}$ & $1.17 \cdot 10^{-4}$ & $1.24 \cdot 10^{-4}$ & $1.32 \cdot 10^{-4}$ & $1.64 \cdot 10^{-4}$ & $1.67 \cdot 10^{-4}$ \\
\hline$k^{(4)}-$ calcium & $2.40 \cdot 10^{-19}$ & $3.01 \cdot 10^{-19}$ & $3.20 \cdot 10^{-19}$ & $3.42 \cdot 10^{-19}$ & $4.35 \cdot 10^{-19}$ & $1.67 \cdot 10^{-4}$ \\
\hline$k^{(5)}-$ bicarbonate & $6.42 \cdot 10^{-3}$ & $6.96 \cdot 10^{-3}$ & $7.10 \cdot 10^{-3}$ & $7.26 \cdot 10^{-3}$ & $7.82 \cdot 10^{-3}$ & $3.30 \cdot 10^{-3}$ \\
\hline$k^{(6)}-$ magnesium & $1.62 \cdot 10^{-4}$ & $1.66 \cdot 10^{-4}$ & $1.68 \cdot 10^{-4}$ & $1.69 \cdot 10^{-4}$ & $1.73 \cdot 10^{-4}$ & $1.67 \cdot 10^{-4}$ \\
\hline$k^{(7)}-$ urea & $1.37 \cdot 10^{-3}$ & $1.39 \cdot 10^{-3}$ & $1.39 \cdot 10^{-3}$ & $1.39 \cdot 10^{-3}$ & $1.41 \cdot 10^{-3}$ & $1.30 \cdot 10^{-3}$ \\
\hline$k^{(8)}-$ creatinine & $1.27 \cdot 10^{-4}$ & $1.28 \cdot 10^{-4}$ & $1.28 \cdot 10^{-4}$ & $1.29 \cdot 10^{-4}$ & $1.30 \cdot 10^{-4}$ & $1.30 \cdot 10^{-4}$ \\
\hline
\end{tabular}

short- and long-term HD complications. ${ }^{29-31}$ The model is based on mass and fluid balance equations, and both fluid and mass exchanges in patient's body compartments and across the dialyzer membrane are taken into account. It can reproduce both the standard hemodialysis (SHD or bicarbonate hemodialysis) and the hemodiafiltration technique (HDF) with pre- (HDF-pre) or post-dilution (HDF-post), by changing the equation of the molar flux across the dialyzer. The body compartments are represented as two pools (intracellular and extracellular) for the mass exchange of solutes, and as three pools (plasmatic, interstitial and intracellular) for the fluid transfer (Figure 1). Symbols in Figure 1 and in the following equations are defined in the Appendix.

Mass, fluid and pressure balance equations that account for mass and fluid exchanges in the body compartments are as follows

$$
\begin{gathered}
\frac{d M_{i c}^{(s)}(t)}{d t}=\phi_{i c}^{(s)}(t) \\
\frac{d M_{e x}^{(s)}(t)}{d t}=-\phi_{i c}^{(s)}(t)-\phi_{\text {dial }}^{(s)}(t)+\phi_{d i l}^{(s)} \\
\frac{d V_{i c}(t)}{d t}=Q_{i c}(t) \\
\frac{d V_{i s}(t)}{d t}=-Q_{i c}(t)+Q_{f c a p}(t) \\
\frac{d V_{p l}(t)}{d t}=-Q_{f c a p}(t)-Q_{u f} \\
\frac{d P_{a c}(t)}{d t}=\frac{1}{C_{c}} \frac{d V_{p l}(t)}{d t} \\
\frac{d P_{i s}(t)}{d t}=E_{i s} \frac{d V_{i s}(t)}{d t}
\end{gathered}
$$

Equations (1) and (2) refer to the mass balance for the intracellular and extracellular compartments, respectively; equations (3) to (5) refer to the fluid balance in the intracellular, interstitial and plasmatic compartments, respectively; and equations (6) and (7) refer to the pressure balance at the arterial capillary side and in the interstitium, respectively. 

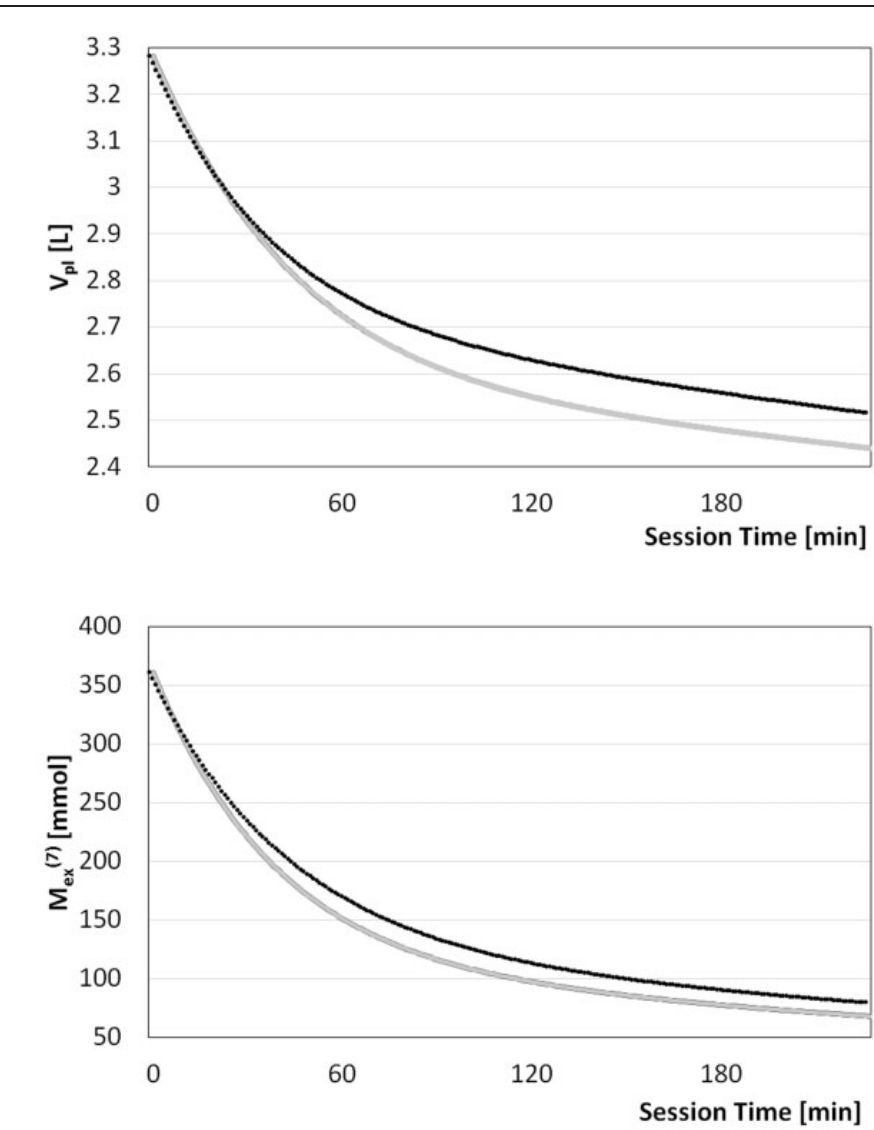

- median value

$2.5 \%$ value

$97.5 \%$ value

..... real

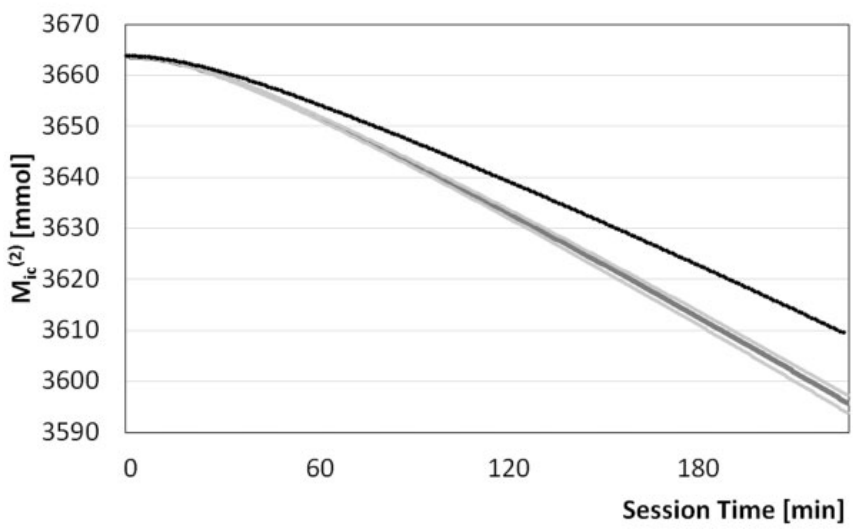

Figure 2. Comparison between simulated and estimated trends for plasmatic volume $V_{p l}(\mathrm{a})$, extracellular mass of urea $M_{\mathrm{ex}}^{(7)}(\mathrm{b})$, and intracellular mass of potassium $M_{i c}^{(2)}$ (c) for HDF-pre in the HFO case.

Individual HD control in terms of mass and fluid exchange is achieved by identifying the patient-specific parameters $\rho, \eta^{(s)}$ and $k^{(s)}$ related to the membranes (see Appendix).

Variables $Q_{\text {fcap }}(t)$ and $\phi_{i c}^{(s)}(t)$ depend on $\rho$ and $k^{(s)}$ as follows

$$
\begin{aligned}
Q_{f c a p}(t) & =\rho L_{c} P_{n}(t) \\
\phi_{i c}^{(s)}(t) & =-k^{(s)}\left(C_{i c}^{(s)}(t)-\beta C_{i s}^{(s)}(t)\right)
\end{aligned}
$$




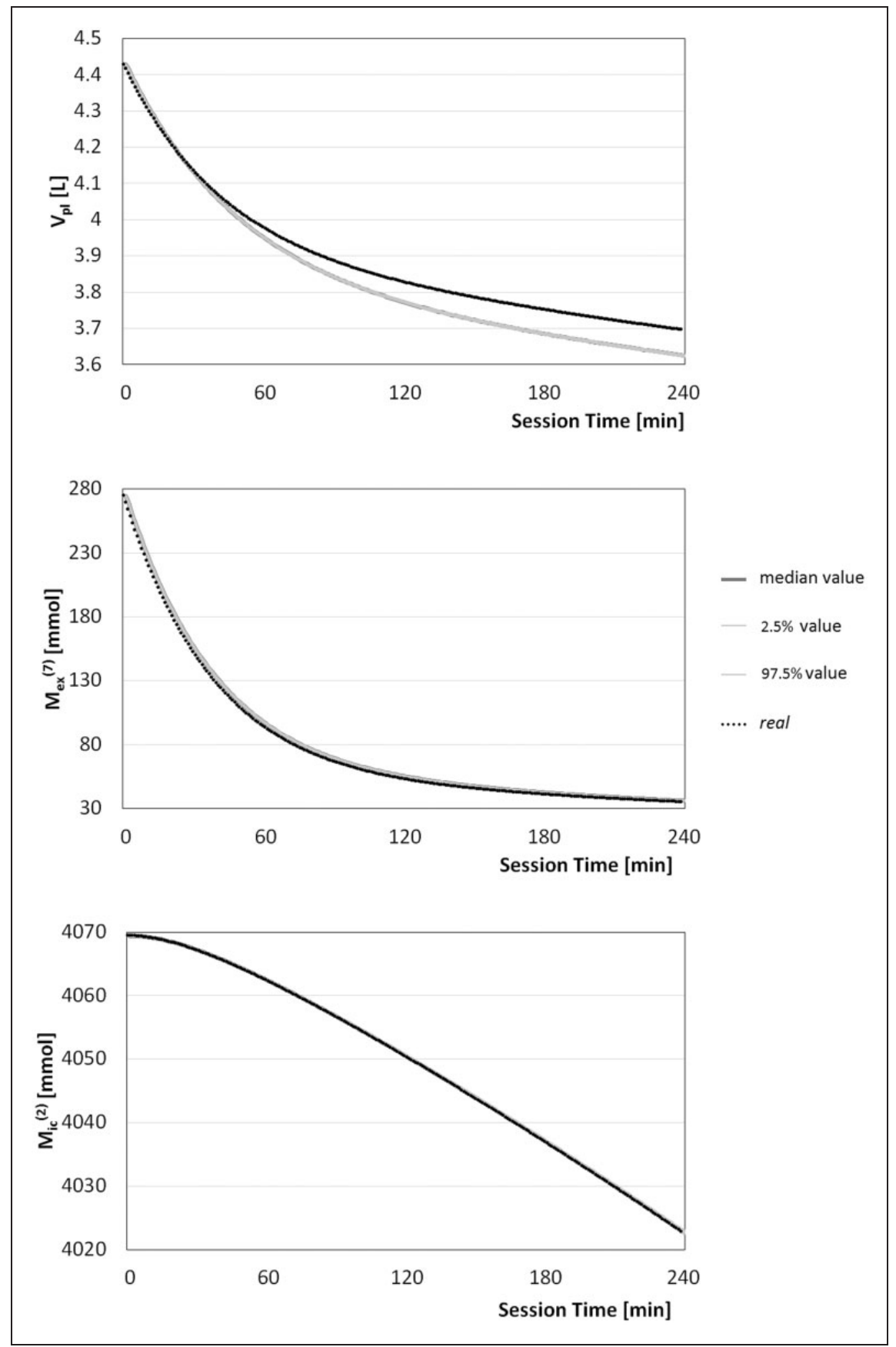

Figure 3. Comparison between simulated and estimated trends for plasmatic volume $V_{p l}(\mathrm{a})$, extracellular mass of urea $M_{\mathrm{ex}}^{(7)}(\mathrm{b})$, and intracellular mass of potassium $M_{i c}^{(2)}$ (c) for SHD in the HFO case.

Variables $\phi_{\text {dial }}^{(s)}(t)$ depend on $\eta^{(s)}$ in different ways, according to the dialysis technique

- Standard hemodialysis (SHD)

$$
\phi_{\text {dial }}^{(s)}(t)=(1-F R) \phi_{\text {diff }}^{(s)}(t)+F R \eta^{(s)} Q_{i n} C_{\text {in }}^{(s)}(t)
$$




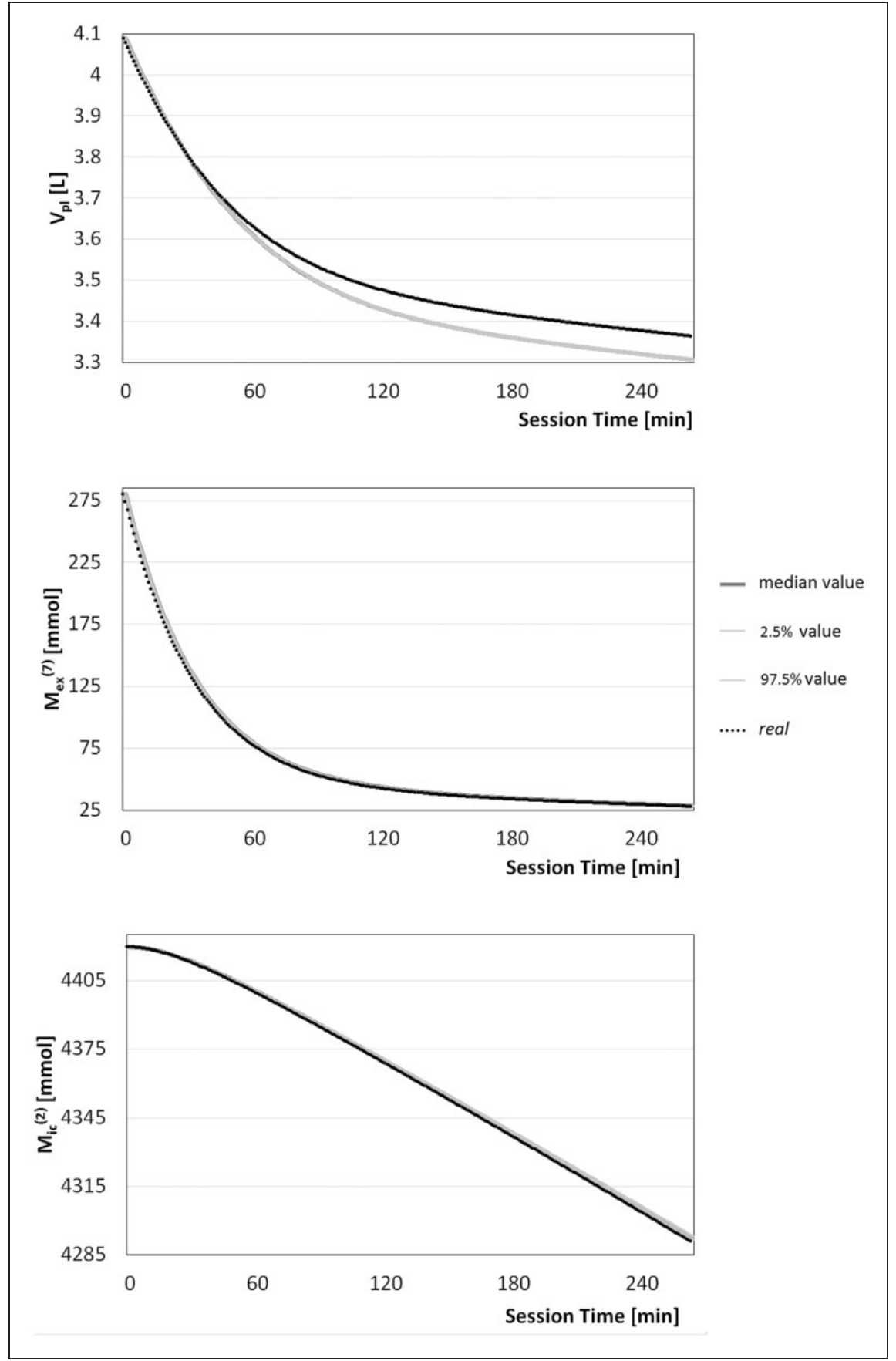

Figure 4. Comparison between simulated and estimated trends for plasmatic volume $V_{p l}(\mathrm{a})$, extracellular mass of urea $M_{\mathrm{ex}}^{(7)}(\mathrm{b})$, and intracellular mass of potassium $M_{i c}^{(2)}$ (c) for HDF-post in the HFO case.

- Hemodiafiltration technique (HDF-pre or HDF-post)

$$
\phi_{\text {dial }}^{(s)}(t)=(1-F R)\left(\phi_{\text {diff }}^{(s)}(t)+\phi_{\text {conv }}^{(s)}(t)\right)+F R\left[Q_{\text {in }}(t)-\left(1-\eta^{(s)}\right) Q_{f}\right] C_{\text {in }}^{(s)}(t)
$$

FR is the filtration ratio, i.e. the plasma water portion filtered by the dialyzer, defined as

$$
F R=\frac{Q_{f}}{Q_{\text {in }}(t)}
$$




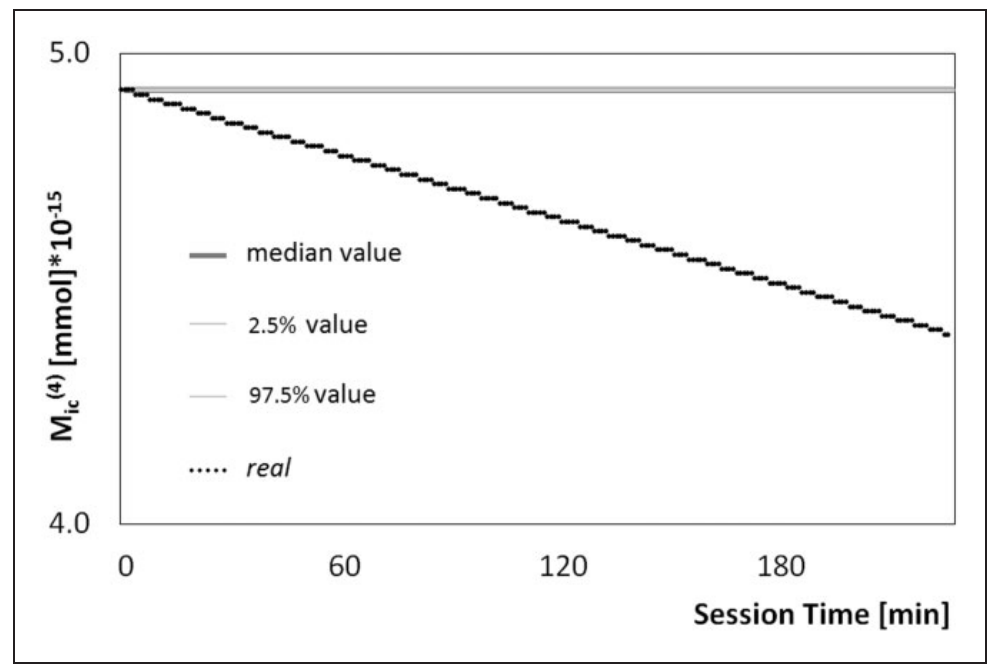

Figure 5. Comparison between simulated and estimated trends for intracellular mass of calcium $M_{i c}^{(4)}$ (c) for HDF-pre in the HFO case.

Table 2. Marginal posterior densities of model parameters vs. their true values (SHD, HFO case).

\begin{tabular}{|c|c|c|c|c|c|c|}
\hline SHD & Min & $25 \%$ & $50 \%$ & $75 \%$ & Max & true \\
\hline$\eta^{(I)}-$ sodium & 1.00 & 1.00 & 1.00 & 1.00 & 1.00 & 1.00 \\
\hline$\eta^{(2)}-$ potassium & 0.95 & 0.99 & 0.99 & 1.00 & 1.00 & 1.00 \\
\hline$\eta^{(3)}-$ chloride & 1.00 & 1.00 & 1.00 & 1.00 & 1.00 & 1.00 \\
\hline$\eta^{(4)}-$ calcium & 1.00 & 1.00 & 1.00 & 1.00 & 1.00 & 1.00 \\
\hline$\eta^{(5)}-$ bicarbonate & 0.00 & 0.00 & 0.00 & 0.01 & 0.05 & 1.00 \\
\hline$\eta^{(6)}-$ magnesium & 0.98 & 1.00 & 1.00 & 1.00 & 1.00 & 1.00 \\
\hline$\eta^{(7)}-$ urea & 0.60 & 0.63 & 0.64 & 0.65 & 0.68 & 1.00 \\
\hline$\eta^{(8)}-$ creatinine & 0.24 & 0.32 & 0.35 & 0.37 & 0.46 & 1.00 \\
\hline$\rho$ & 0.84 & 0.85 & 0.85 & 0.84 & 0.85 & 1.00 \\
\hline$k^{(1)}-$ sodium & $7.23 \cdot 10^{-4}$ & $8.38 \cdot 10^{-3}$ & $8.65 \cdot 10^{-4}$ & $8.89 \cdot 10^{-4}$ & $9.72 \cdot 10^{-4}$ & $2.50 \cdot 10^{-3}$ \\
\hline$k^{(2)}-$ potassium & $1.69 \cdot 10^{-4}$ & $1.70 \cdot 10^{-4}$ & $1.71 \cdot 10^{-4}$ & $1.71 \cdot 10^{-4}$ & $1.72 \cdot 10^{-4}$ & $1.67 \cdot 10^{-4}$ \\
\hline$k^{(3)}-$ chloride & $1.65 \cdot 10^{-4}$ & $2.01 \cdot 10^{-4}$ & $2.13 \cdot 10^{-4}$ & $2.25 \cdot 10^{-4}$ & $2.80 \cdot 10^{-4}$ & $1.67 \cdot 10^{-4}$ \\
\hline$k^{(4)}-$ calcium & $2.21 \cdot 10^{-19}$ & $2.21 \cdot 10^{-19}$ & $2.21 \cdot 10^{-19}$ & $2.21 \cdot 10^{-19}$ & $2.21 \cdot 10^{-19}$ & $1.67 \cdot 10^{-4}$ \\
\hline$k^{(5)}$ - bicarbonate & $6.75 \cdot 10^{-3}$ & $6.91 \cdot 10^{-3}$ & $6.94 \cdot 10^{-3}$ & $6.96 \cdot 10^{-3}$ & $7.02 \cdot 10^{-3}$ & $3.30 \cdot 10^{-3}$ \\
\hline$k^{(6)}-$ magnesium & $1.67 \cdot 10^{-4}$ & $1.73 \cdot 10^{-4}$ & $1.74 \cdot 10^{-4}$ & $1.76 \cdot 10^{-4}$ & $1.82 \cdot 10^{-4}$ & $1.67 \cdot 10^{-4}$ \\
\hline$k^{(7)}-$ urea & $1.36 \cdot 10^{-3}$ & $1.37 \cdot 10^{-3}$ & $1.37 \cdot 10^{-3}$ & $1.37 \cdot 10^{-3}$ & $1.37 \cdot 10^{-3}$ & $1.30 \cdot 10^{-3}$ \\
\hline$k^{(8)}-$ creatinine & $1.30 \cdot 10^{-4}$ & $1.31 \cdot 10^{-4}$ & $1.31 \cdot 10^{-4}$ & $1.31 \cdot 10^{-4}$ & $1.32 \cdot 10^{-4}$ & $1.30 \cdot 10^{-4}$ \\
\hline
\end{tabular}

and $Q_{\text {in }}(t)$ is the plasma water flow rate at the dialyzer inlet, which corresponds to the prescribed blood flow rate disregarding the red blood cells and the protein component contributions (plasma water fraction according to Colton et al. $^{32}$ ). As for HDF-post and SHD, $Q_{\text {in }}$ is expressed as

$$
Q_{\text {in }}(t)=Q_{b}\left(1-\frac{H t(t)}{100}\right)\left(1-0.0107 T_{p}(t)\right)
$$

while for HDF-pre, it is incremented by the dilution flow rate as follows

$$
Q_{i n}(t)=Q_{b}\left(1-\frac{H t(t)}{100}\right)\left(1-0.0107 T_{p}(t)\right)+Q_{d i l}
$$




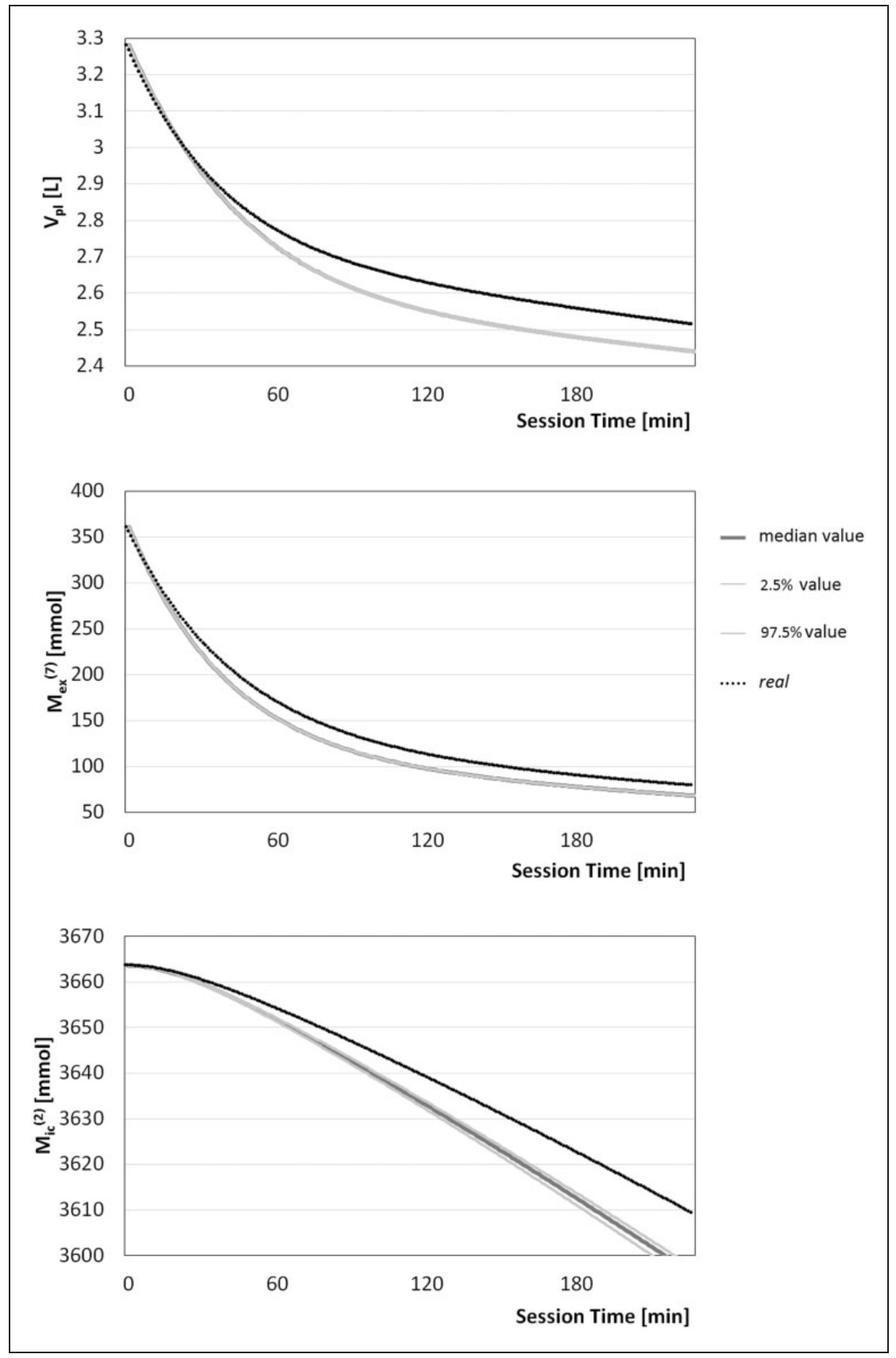

Figure 6. Comparison between simulated and estimated trends for plasmatic volume $V_{p l}(\mathrm{a})$, extracellular mass of urea $M_{\mathrm{ex}}^{(7)}(\mathrm{b})$, and intracellular mass of potassium $M_{i c}^{(2)}$ (c) for HDF-pre in the HFO case - cross check of parameter $k^{(4)}$.

\section{Bayesian estimation approach}

Let us denote by $\Theta=\left\{\eta^{(1)}, \ldots, \eta^{(8)}, \rho, k^{(1)}, \ldots, k^{(8)}\right\}$ the set of the 17 patient-specific parameters to estimate, and by $\hat{\mathbf{X}}_{o b s}$ the set of the available observations for the state variables in equations (1) to (7).

In the Bayesian setting, we obtain the posterior densities of the parameters $f\left(\Theta \mid \hat{\mathbf{X}}_{o b s}\right)$ given the likelihood function $f\left(\hat{\mathbf{X}}_{o b s} \mid \Theta\right)$ and the prior density $f(\Theta)$ of the parameters. Both ingredients are detailed below. Given the structure of the model, the posterior densities are numerically obtained with a Markov Chain Monte Carlo (MCMC) algorithm. 
Table 3. Marginal posterior densities of model parameters vs. their true values (HDF-post, HFO case).

\begin{tabular}{lllllll}
\hline HDF-post & Min & $25 \%$ & $50 \%$ & $75 \%$ & Max & true \\
\hline$\eta^{(1)}-$ sodium & 0.52 & 0.52 & 0.53 & 0.53 & 0.53 & 0.50 \\
$\eta^{(2)}-$ potassium & 0.45 & 0.46 & 0.47 & 0.47 & 0.49 & 0.50 \\
$\eta^{(3)}-$ chloride & 0.52 & 0.52 & 0.52 & 0.52 & 0.53 & 0.50 \\
$\eta^{(4)}-$ calcium & 0.52 & 0.52 & 0.53 & 0.53 & 0.53 & 0.50 \\
$\eta^{(5)}-$ bicarbonate & 0.52 & 0.52 & 0.52 & 0.52 & 0.53 & 0.50 \\
$\eta^{(6)}-$ magnesium & 0.47 & 0.48 & 0.48 & 0.49 & 0.50 & 0.50 \\
$\eta^{(7)}-$ urea & 0.46 & 0.46 & 0.46 & 0.46 & 0.47 & 0.50 \\
$\eta^{(8)}-$ creatinine & 0.38 & 0.39 & 0.40 & 0.40 & 0.42 & 0.50 \\
$\rho$ & 0.81 & 0.82 & 0.82 & 0.82 & 0.83 & 1.00 \\
$k^{(1)}-$ sodium & $2.92 \cdot 10^{-3}$ & $3.02 \cdot 10^{-3}$ & $3.04 \cdot 10^{-3}$ & $3.07 \cdot 10^{-3}$ & $3.16 \cdot 10^{-3}$ & $2.50 \cdot 10^{-3}$ \\
$k^{(2)}-$ potassium & $1.63 \cdot 10^{-4}$ & $1.63 \cdot 10^{-4}$ & $1.63 \cdot 10^{-4}$ & $1.63 \cdot 10^{-4}$ & $1.63 \cdot 10^{-4}$ & $1.67 \cdot 10^{-4}$ \\
$k^{(3)}-$ chloride & $9.93 \cdot 10^{-5}$ & $1.23 \cdot 10^{-4}$ & $1.30 \cdot 10^{-4}$ & $1.39 \cdot 10^{-4}$ & $1.79 \cdot 10^{-4}$ & $1.67 \cdot 10^{-4}$ \\
$k^{(4)}-$ calcium & $1.24 \cdot 10^{-19}$ & $1.77 \cdot 10^{-19}$ & $1.89 \cdot 10^{-19}$ & $2.03 \cdot 10^{-18}$ & $2.68 \cdot 10^{-19}$ & $1.67 \cdot 10^{-4}$ \\
$k^{(5)}-$ bicarbonate & $6.81 \cdot 10^{-4}$ & $7.72 \cdot 10^{-3}$ & $8.00 \cdot 10^{-4}$ & $8.26 \cdot 10^{-4}$ & $9.47 \cdot 10^{-4}$ & $3.30 \cdot 10^{-3}$ \\
$k^{(6)}-$ magnesium & $1.63 \cdot 10^{-4}$ & $1.64 \cdot 10^{-4}$ & $1.65 \cdot 10^{-4}$ & $1.65 \cdot 10^{-4}$ & $1.67 \cdot 10^{-4}$ & $1.67 \cdot 10^{-4}$ \\
$k^{(7)}-$ urea & $1.33 \cdot 10^{-3}$ & $1.34 \cdot 10^{-3}$ & $1.34 \cdot 10^{-3}$ & $1.34 \cdot 10^{-3}$ & $1.34 \cdot 10^{-3}$ & $1.30 \cdot 10^{-3}$ \\
$k^{(8)}-$ creatinine & $1.28 \cdot 10^{-4}$ & $1.28 \cdot 10^{-4}$ & $1.28 \cdot 10^{-4}$ & $1.28 \cdot 10^{-4}$ & $1.29 \cdot 10^{-4}$ & $1.30 \cdot 10^{-4}$ \\
\hline
\end{tabular}

Table 4. Marginal posterior densities of model parameters vs. their true values (HDF-pre, LFO case).

\begin{tabular}{lllllll}
\hline Parameter & Min & $25 \%$ & $50 \%$ & $75 \%$ & Max & true \\
\hline$\eta^{(1)}-$ sodium & 0.06 & 0.12 & 0.14 & 0.16 & 0.23 & 0.50 \\
$\eta^{(2)}-$ potassium & 0.77 & 0.95 & 0.97 & 0.99 & 1.00 & 0.50 \\
$\eta^{(3)}-$ chloride & 0.69 & 0.86 & 0.92 & 0.96 & 1.00 & 0.50 \\
$\eta^{(4)}-$ calcium & 0.00 & 0.50 & 0.67 & 0.79 & 1.00 & 0.50 \\
$\eta^{(5)}-$ bicarbonate & 0.89 & 0.97 & 0.98 & 0.99 & 1.00 & 0.50 \\
$\eta^{(6)}-$ magnesium & 0.06 & 0.74 & 0.86 & 0.94 & 1.00 & 0.50 \\
$\eta^{(7)}-$ urea & 0.90 & 0.97 & 0.99 & 0.99 & 1.00 & 0.50 \\
$\eta^{(8)}-$ creatinine & 0.01 & 0.59 & 0.77 & 0.89 & 1.00 & 0.50 \\
$\rho$ & 0.87 & 0.88 & 0.88 & 0.88 & 0.89 & 1.00 \\
$k^{(1)}-$ sodium & $1.83 \cdot 10^{-3}$ & $2.18 \cdot 10^{-3}$ & $2.29 \cdot 10^{-3}$ & $2.42 \cdot 10^{-3}$ & $2.84 \cdot 10^{-3}$ & $2.50 \cdot 10^{-3}$ \\
$k^{(2)}-$ potassium & $1.14 \cdot 10^{-4}$ & $1.58 \cdot 10^{-4}$ & $1.69 \cdot 10^{-4}$ & $1.80 \cdot 10^{-4}$ & $2.30 \cdot 10^{-4}$ & $1.67 \cdot 10^{-4}$ \\
$k^{(3)}-$ chloride & $1.12 \cdot 10^{-4}$ & $1.53 \cdot 10^{-4}$ & $1.64 \cdot 10^{-4}$ & $1.75 \cdot 10^{-4}$ & $2.33 \cdot 10^{-4}$ & $1.67 \cdot 10^{-4}$ \\
$k^{(4)}-$ calcium & $1.19 \cdot 10^{-4}$ & $1.54 \cdot 10^{-4}$ & $1.64 \cdot 10^{-4}$ & $1.75 \cdot 10^{-4}$ & $2.21 \cdot 10^{-4}$ & $1.67 \cdot 10^{-4}$ \\
$k^{(5)}-$ bicarbonate & $2.55 \cdot 10^{-3}$ & $3.24 \cdot 10^{-3}$ & $3.43 \cdot 10^{-3}$ & $3.66 \cdot 10^{-3}$ & $4.56 \cdot 10^{-3}$ & $3.30 \cdot 10^{-3}$ \\
$k^{(6)}-$ magnesium & $1.31 \cdot 10^{-4}$ & $1.58 \cdot 10^{-4}$ & $1.68 \cdot 10^{-4}$ & $1.77 \cdot 10^{-4}$ & $2.12 \cdot 10^{-4}$ & $1.67 \cdot 10^{-4}$ \\
$k^{(7)}-$ urea & $1.20 \cdot 10^{-3}$ & $1.45 \cdot 10^{-3}$ & $1.52 \cdot 10^{-3}$ & $1.61 \cdot 10^{-3}$ & $1.91 \cdot 10^{-3}$ & $1.30 \cdot 10^{-3}$ \\
$k^{(8)}-$ creatinine & $9.15 \cdot 10^{-5}$ & $1.23 \cdot 10^{-4}$ & $1.30 \cdot 10^{-4}$ & $1.40 \cdot 10^{-4}$ & $1.79 \cdot 10^{-4}$ & $1.30 \cdot 10^{-4}$ \\
\hline
\end{tabular}

\section{I Likelihood function}

The likelihood function $f\left(\hat{\mathbf{X}}_{\text {obs }} \mid \Theta\right)$ is based on a discretized version of the multi-compartment model.

We consider an explicit discretization of the ODE system, in which a value $X^{i}\left(t_{h}\right)$ is computed for each state variable $i(i=1, \ldots, n)$ at each time instant $t_{h}$ based on the values of all state variables at the previous instant $t_{h-1}$. To guarantee the convergence, the discretization is performed with the $4^{\text {th }}$-order Runge-Kutta method.

Each patient-specific parameter in $\Theta$ is assumed to be a random variable. In this way, each discretized equation is a random process, and we may express the density of each $X^{i}\left(t_{h}\right)$ as conditioned to $\Theta$ and to all values $X^{1}\left(t_{h-1}\right), \ldots, X^{n}\left(t_{h-1}\right)$ at the previous time instant $t_{h-1}$ :

$$
X^{i}\left(t_{h}\right) \sim \mathcal{L}\left(X^{i}\left(t_{h}\right) \mid X^{1}\left(t_{h-1}\right), \ldots, X^{n}\left(t_{h-1}\right), \Theta\right) \quad \forall i, h
$$


Table 5. Marginal posterior densities of model parameters vs. their true values (SHD, LFO case).

\begin{tabular}{|c|c|c|c|c|c|c|}
\hline Parameter & Min & $25 \%$ & $50 \%$ & $75 \%$ & Max & true \\
\hline$\eta^{(\mathrm{I})}-$ sodium & 0.81 & 0.96 & 0.98 & 0.99 & 1.00 & 1.00 \\
\hline$\eta^{(2)}-$ potassium & 0.01 & 0.71 & 0.84 & 0.92 & 1.00 & 1.00 \\
\hline$\eta^{(3)}-$ chloride & 0.50 & 0.91 & 0.95 & 0.98 & 1.00 & 1.00 \\
\hline$\eta^{(4)}-$ calcium & 0.01 & 0.68 & 0.84 & 0.94 & 1.00 & 1.00 \\
\hline$\eta^{(5)}-$ bicarbonate & 0.44 & 0.88 & 0.94 & 0.97 & 1.00 & 1.00 \\
\hline$\eta^{(6)}-$ magnesium & 0.02 & 0.54 & 0.75 & 0.88 & 1.00 & 1.00 \\
\hline$\eta^{(7)}-$ urea & 0.23 & 0.80 & 0.90 & 0.95 & 1.00 & 1.00 \\
\hline$\eta^{(8)}-$ creatinine & 0.00 & 0.31 & 0.54 & 0.80 & 1.00 & 1.00 \\
\hline$\rho$ & 0.82 & 0.83 & 0.83 & 0.83 & 0.84 & 1.00 \\
\hline$k^{(1)}-$ sodium & $1.74 \cdot 10^{-3}$ & $2.24 \cdot 10^{-3}$ & $2.41 \cdot 10^{-3}$ & $2.58 \cdot 10^{-3}$ & $3.23 \cdot 10^{-3}$ & $2.50 \cdot 10^{-3}$ \\
\hline$k^{(2)}-$ potassium & $1.21 \cdot 10^{-4}$ & $1.48 \cdot 10^{-4}$ & $1.57 \cdot 10^{-4}$ & $1.66 \cdot 10^{-4}$ & $2.02 \cdot 10^{-4}$ & $1.67 \cdot 10^{-4}$ \\
\hline$k^{(3)}-$ chloride & $1.24 \cdot 10^{-4}$ & $1.54 \cdot 10^{-4}$ & $3.29 \cdot 10^{-3}$ & $1.76 \cdot 10^{-4}$ & $1.46 \cdot 10^{-3}$ & $1.67 \cdot 10^{-4}$ \\
\hline$k^{(4)}-$ calcium & $1.18 \cdot 10^{-4}$ & $1.18 \cdot 10^{-4}$ & $1.18 \cdot 10^{-4}$ & $1.18 \cdot 10^{-4}$ & $1.18 \cdot 10^{-4}$ & $1.67 \cdot 10^{-4}$ \\
\hline$k^{(5)}-$ bicarbonate & $2.31 \cdot 10^{-3}$ & $3.08 \cdot 10^{-3}$ & $3.29 \cdot 10^{-3}$ & $3.51 \cdot 10^{-3}$ & $4.17 \cdot 10^{-3}$ & $3.30 \cdot 10^{-3}$ \\
\hline$k^{(6)}-$ magnesium & $1.06 \cdot 10^{-4}$ & $1.55 \cdot 10^{-4}$ & $1.65 \cdot 10^{-4}$ & $1.76 \cdot 10^{-4}$ & $2.18 \cdot 10^{-4}$ & $1.67 \cdot 10^{-4}$ \\
\hline$k^{(7)}-$ urea & $1.12 \cdot 10^{-3}$ & $1.30 \cdot 10^{-3}$ & $1.34 \cdot 10^{-3}$ & $1.37 \cdot 10^{-3}$ & $1.46 \cdot 10^{-3}$ & $1.30 \cdot 10^{-3}$ \\
\hline$k^{(8)}-$ creatinine & $1.08 \cdot 10^{-4}$ & $1.28 \cdot 10^{-4}$ & $1.31 \cdot 10^{-4}$ & $1.34 \cdot 10^{-4}$ & $1.55 \cdot 10^{-4}$ & $1.30 \cdot 10^{-4}$ \\
\hline
\end{tabular}

Table 6. Marginalposterior densities of model parameters vs. their true values (HDF-post, LFO case).

\begin{tabular}{|c|c|c|c|c|c|c|}
\hline Parameter & Min & $25 \%$ & $50 \%$ & $75 \%$ & Max & true \\
\hline$\eta^{(1)}-$ sodium & 0.73 & 0.94 & 0.97 & 0.99 & 1.00 & 1.00 \\
\hline$\eta^{(2)}-$ potassium & 0.05 & 0.82 & 0.91 & 0.96 & 1.00 & 1.00 \\
\hline$\eta^{(3)}-$ chloride & 0.00 & 0.24 & 0.35 & 0.47 & 0.91 & 1.00 \\
\hline$\eta^{(4)}-$ calcium & 0.01 & 0.39 & 0.55 & 0.72 & 0.99 & 1.00 \\
\hline$\eta^{(5)}-$ bicarbonate & 0.36 & 0.81 & 0.90 & 0.95 & 1.00 & 1.00 \\
\hline$\eta^{(6)}-$ magnesium & 0.01 & 0.46 & 0.68 & 0.84 & 1.00 & 1.00 \\
\hline$\eta^{(7)}-$ urea & 0.68 & 0.95 & 0.97 & 0.99 & 1.00 & 1.00 \\
\hline$\eta^{(8)}-$ creatinine & 0.00 & 0.39 & 0.67 & 0.87 & 1.00 & 1.00 \\
\hline$\rho$ & 0.82 & 0.83 & 0.83 & 0.83 & 0.84 & 1.00 \\
\hline$k^{(1)}-$ sodium & $1.92 \cdot 10^{-3}$ & $2.42 \cdot 10^{-3}$ & $2.59 \cdot 10^{-3}$ & $2.76 \cdot 10^{-3}$ & $3.55 \cdot 10^{-3}$ & $2.50 \cdot 10^{-3}$ \\
\hline$k^{(2)}-$ potassium & $1.24 \cdot 10^{-4}$ & $1.24 \cdot 10^{-4}$ & $1.24 \cdot 10^{-4}$ & $1.24 \cdot 10^{-4}$ & $1.24 \cdot 10^{-4}$ & $1.67 \cdot 10^{-4}$ \\
\hline$k^{(3)}-$ chloride & $1.17 \cdot 10^{-4}$ & $1.54 \cdot 10^{-4}$ & $1.67 \cdot 10^{-4}$ & $1.78 \cdot 10^{-4}$ & $2.35 \cdot 10^{-4}$ & $1.67 \cdot 10^{-4}$ \\
\hline$k^{(4)}-$ calcium & $1.21 \cdot 10^{-4}$ & $1.54 \cdot 10^{-4}$ & $1.65 \cdot 10^{-4}$ & $1.76 \cdot 10^{-4}$ & $2.17 \cdot 10^{-4}$ & $1.67 \cdot 10^{-4}$ \\
\hline$k^{(5)}$ - bicarbonate & $2.22 \cdot 10^{-3}$ & $2.92 \cdot 10^{-3}$ & $3.14 \cdot 10^{-3}$ & $3.36 \cdot 10^{-3}$ & $4.34 \cdot 10^{-3}$ & $3.30 \cdot 10^{-3}$ \\
\hline$k^{(6)}-$ magnesium & $1.21 \cdot 10^{-4}$ & $1.58 \cdot 10^{-4}$ & $1.69 \cdot 10^{-4}$ & $1.80 \cdot 10^{-4}$ & $2.36 \cdot 10^{-4}$ & $1.67 \cdot 10^{-4}$ \\
\hline$k^{(7)}-$ urea & $1.16 \cdot 10^{-3}$ & $1.46 \cdot 10^{-3}$ & $1.54 \cdot 10^{-3}$ & $1.61 \cdot 10^{-3}$ & $1.95 \cdot 10^{-3}$ & $1.30 \cdot 10^{-3}$ \\
\hline$k^{(8)}-$ creatinine & $1.03 \cdot 10^{-4}$ & $1.24 \cdot 10^{-4}$ & $1.31 \cdot 10^{-4}$ & $1.39 \cdot 10^{-4}$ & $1.66 \cdot 10^{-4}$ & $1.30 \cdot 10^{-4}$ \\
\hline
\end{tabular}

where $\mathcal{L}$ denotes the conditioned probability law, which does not follow any known form due to the structure of the system.

We now consider that observations $X_{o b s}^{i} \in \hat{\mathbf{X}}_{o b s}$ of state variables $X^{i}$ are available only at some time instants $t_{h^{*}}$, where the set $\left\{t_{h^{*}}\right\}$ in which observations are available is a subset of all time instants $\left\{t_{h}\right\}$. This is because the discretization step $\Delta$, chosen according to the differential equations, is usually thicker than the frequency of the observations.

Moreover, we assume that all observations $X_{o b s}^{i}\left(t_{h^{*}}\right)$ are associated with an error (e.g. a measurement error). Thus, we model them as stochastic variables centered on the value computed from the model. We consider a Gamma distribution, to respect the positivity of the variables, with modal value $X^{i}\left(t_{h^{*}}\right)$ and standard deviation $\alpha X^{i}\left(t_{h^{*}}\right)$

$$
X_{o b s}^{i}\left(t_{h^{*}}\right) \sim \mathcal{G}\left(1+\frac{1+\sqrt{1+4 \alpha^{2}}}{2 \alpha^{2}}, \frac{1+\sqrt{1+4 \alpha^{2}}}{2 \alpha^{2} X^{i}\left(t_{h^{*}}\right)}\right) \quad \forall i, h^{*}
$$


Table 7. Registry data (gender and age), initial weight at the beginning of the HD session, and therapy prescription (session time and ultrafiltration) for the three tested patients.

\begin{tabular}{llll}
\hline Technique & HDF-pre & SHD & HDF-post \\
\hline Gender & $\mathrm{F}$ & $\mathrm{M}$ & $\mathrm{M}$ \\
Age (years) & 50 & 74 & 64 \\
Initial weight (kg) & 63.6 & 81 & 77.3 \\
Session Time (h) & 4 & 4 & 4.5 \\
Ultrafiltration (L) & 3 & 3.2 & 3.1 \\
\hline
\end{tabular}

Table 8. Time-dependent variables for the three tested patients with the respective frequency.

\begin{tabular}{lc}
\hline Variable & Frequency \\
\hline Plasma sodium concentration $C_{p l}^{(I)}(t)(\mathrm{mmol} / \mathrm{L})$ & $\begin{array}{c}\text { Beginning, ending, and every hour } \\
\text { during the treatment }\end{array}$ \\
Plasma potassium concentration $C_{p l}^{(2)}(t)(\mathrm{mmol} / \mathrm{L})$ & \\
Plasma chloride concentration $C_{p l}^{(3)}(t)(\mathrm{mmol} / \mathrm{L})$ & \\
Plasma calcium concentration $C_{p l}^{(4)}(t)(\mathrm{mmol} / \mathrm{L})$ & \\
Plasma bicarbonate concentration $C_{p l}^{(5)}(t)(\mathrm{mmol} / \mathrm{L})$ & beginning and ending of the treatment \\
Plasma urea concentration $C_{p l}^{(7)}(t)(\mathrm{mmol} / \mathrm{L})$ & \\
Hematocrit $H t(t)(\%)$ & every minute \\
Plasma albumin concentration $[a l b u m i n](t)(\mathrm{g} / \mathrm{L})$ & \\
Plasma magnesium concentration $C_{p l}^{(6)}(t)(\mathrm{mmol} / \mathrm{L})$ & \\
Plasma creatinine concentration $C_{p l}^{(8)}(t)(\mathrm{mmol} / \mathrm{L})$ & \\
Relative volume $R B V(t)(\%)$ & \\
Ultrafiltration volume $U F V(t)(L)$ &
\end{tabular}

where $\mathcal{G}$ denotes the Gamma distribution, $1+\frac{1+\sqrt{1+4 \alpha^{2}}}{2 \alpha^{2}}$ is the shape parameter, and $\frac{1+\sqrt{1+4 \alpha^{2}}}{2 \alpha^{2} X^{i}\left(t_{h^{*}}\right)}$ the rate parameter.

The choice of a probability density function centered on the value computed from the model is common in this type of approach. More specifically, the chosen Gamma density respects the characteristics of the problem, i.e. the positive support of the density respects the positiveness of the variable, and fitting the modal value with the model allows to have a distribution with a peak. Such choice has been already adopted and gave fair results. ${ }^{28}$

In particular, we use three different parameters $\alpha$ based on the state variable, i.e. $\alpha_{v}$ for $V_{i s}$ and $V_{p l}, \alpha_{m e x}$ for all $M_{e x}^{(s)}$, and $\alpha_{m i c}$ for $M_{i c}^{(s)}$. They are other parameters to estimate; thus, the overall set of the parameters to estimate $\Theta^{*}=\Theta \cup\left\{\alpha_{v}, \alpha_{\text {mex }}, \alpha_{\text {mic }}\right\}$ includes 20 elements.

The combination of equations (8) and (9) gives the conditional law of each observation at each instant $t_{h^{*}}$. Their product over $t_{h^{*}}$ and $i$ gives the likelihood function $f\left(\hat{\mathbf{X}}_{o b s} \mid \Theta^{*}\right)$ of the observations given the overall parameter vector $\Theta^{*}$

$$
\begin{aligned}
f\left(\hat{\mathbf{X}}_{o b s} \mid \Theta^{*}\right)= & \prod_{i, t_{h^{*}}} \mathcal{G}\left(1+\frac{1+\sqrt{1+4 \alpha^{2}}}{2 \alpha^{2}}, \frac{1+\sqrt{1+4 \alpha^{2}}}{2 \alpha^{2} X^{i}\left(t_{h^{*}}\right)}\right) \\
& \times \hat{\mathcal{L}}\left(X^{i}\left(t_{h^{*}}\right) \mid X^{1}\left(t_{h^{*}-1}\right), \ldots, X^{n}\left(t_{h^{*}-1}\right), \Theta^{*}\right)
\end{aligned}
$$

The law $\hat{\mathcal{L}}$ is the marginal density of $X^{i}\left(t_{h^{*}}\right)$ given $\Theta^{*}$ and the observations at the previous $t_{h^{*}-1}$; it is derived combining all of the densities $\mathcal{L}$ in equation (8) for all variables between $t_{h^{*}-1}$ and $t_{h^{*}}$. In the notation, $\alpha$ has to be specified $\left(\alpha_{v}, \alpha_{\text {mex }}\right.$ or $\left.\alpha_{\text {mic }}\right)$ based on the specific variable $X^{i}$. 
Table 9. Marginal posterior densities of model parameters (HDF-pre, real patient).

\begin{tabular}{|c|c|c|c|c|c|}
\hline Parameter & Min & $25 \%$ & $50 \%$ & $75 \%$ & Max \\
\hline$\eta^{(1)}-$ sodium & 0.41 & 0.58 & 0.64 & 0.69 & 0.84 \\
\hline$\eta^{(2)}-$ potassium & 0.29 & 0.84 & 0.91 & 0.96 & 1.00 \\
\hline$\eta^{(3)}-$ chloride & 0.09 & 0.28 & 0.33 & 0.38 & 0.58 \\
\hline$\eta^{(4)}-$ calcium & 0.00 & 0.47 & 0.59 & 0.69 & 0.99 \\
\hline$\eta^{(5)}-$ bicarbonate & 0.30 & 0.71 & 0.82 & 0.90 & 1.00 \\
\hline$\eta^{(6)}-$ magnesium & 0.02 & 0.42 & 0.66 & 0.84 & 1.00 \\
\hline$\eta^{(7)}-$ urea & 0.59 & 0.93 & 0.96 & 0.98 & 1.00 \\
\hline$\eta^{(8)}-$ creatinine & 0.01 & 0.38 & 0.62 & 0.83 & 1.00 \\
\hline$\rho$ & 0.71 & 0.73 & 0.73 & 0.73 & 0.75 \\
\hline$k^{(1)}-$ sodium & $1.66 \cdot 10^{-3}$ & $2.30 \cdot 10^{-3}$ & $2.47 \cdot 10^{-3}$ & $2.65 \cdot 10^{-3}$ & $3.37 \cdot 10^{-3}$ \\
\hline$k^{(2)}-$ potassium & $1.22 \cdot 10^{-4}$ & $1.57 \cdot 10^{-4}$ & $1.68 \cdot 10^{-4}$ & I.79.1 $10^{-4}$ & $2.28 \cdot 10^{-4}$ \\
\hline$k^{(3)}-$ chloride & $1.20 \cdot 10^{-4}$ & $1.56 \cdot 10^{-4}$ & $1.68 \cdot 10^{-4}$ & $1.80 \cdot 10^{-4}$ & $2.37 \cdot 10^{-4}$ \\
\hline$k^{(4)}-$ calcium & $1.20 \cdot 10^{-4}$ & $1.55 \cdot 10^{-4}$ & $1.65 \cdot 10^{-4}$ & $1.76 \cdot 10^{-4}$ & $2.22 \cdot 10^{-4}$ \\
\hline$k^{(5)}-$ bicarbonate & $2.26 \cdot 10^{-3}$ & $3.02 \cdot 10^{-3}$ & $3.27 \cdot 10^{-3}$ & $3.48 \cdot 10^{-3}$ & $4.35 \cdot 10^{-3}$ \\
\hline$k^{(6)}-$ magnesium & $1.18 \cdot 10^{-4}$ & $1.56 \cdot 10^{-4}$ & $1.66 \cdot 10^{-4}$ & $1.78 \cdot 10^{-4}$ & $2.28 \cdot 10^{-4}$ \\
\hline$k^{(7)}-$ urea & $9.80 \cdot 10^{-4}$ & $1.29 \cdot 10^{-3}$ & $1.38 \cdot 10^{-3}$ & $1.48 \cdot 10^{-3}$ & $1.87 \cdot 10^{-3}$ \\
\hline$k^{(8)}-$ creatinine & $9.39 \cdot 10^{-5}$ & $1.22 \cdot 10^{-4}$ & $1.30 \cdot 10^{-4}$ & $1.39 \cdot 10^{-4}$ & $1.78 \cdot 10^{-4}$ \\
\hline
\end{tabular}

To make simpler the notation, we did not mention above that the discretization step $\Delta$ can be different from variable to variable, so that the observations of all variables are available at some instants $t_{h^{*}}$ while only a subset of observations in other instants $t_{h^{*}}$. However, this additional feature does not change the approach, while it only makes the notation for $\hat{\mathcal{L}}$ more complicated.

\subsection{Prior densities and computation of the posterior densities}

We assume that all parameters in $\Theta^{*}$ are a priori independent. According to the HD literature, ${ }^{9,33}$ which provides some information about standard values and ranges, the following prior densities are chosen for the parameters in $\Theta$ :

- $\eta^{(s)}$ : Uniform density with minimum value equal to 0 and maximum value equal to $1 \forall s$;

- $\rho$ : Gamma density with mean value equal to 1 and standard deviation equal to 0.1 ;

- $k^{(s)}$ : Gamma density with mean value equal to $2.50 \cdot 10^{-3} \mathrm{~L} / \mathrm{sec}$ for sodium, $3.30 \cdot 10^{-3} \mathrm{~L} / \mathrm{sec}$ for bicarbonate, $1.30 \cdot 10^{-3} \mathrm{~L} / \mathrm{sec}$ for urea, $1.30 \cdot 10^{-4} \mathrm{~L} / \mathrm{sec}$ for creatinine, $1.67 \cdot 10^{-4} \mathrm{~L} / \mathrm{sec}$ for all other solutes, and standard deviation equal to the $10 \%$ of the respective mean value $\forall s$.

For the additional parameters $\alpha_{v}, \alpha_{m e x}$ and $\alpha_{m i c}$ in $\Theta^{*}$, Gamma densities with both mean value and variance equal to 0.05 are considered.

The posterior density of $\Theta^{*}$ is numerically obtained with a Markov Chain Monte Carlo (MCMC) method. The model has been implemented in STAN ${ }^{34}$ through its R interface, which implements the Hamiltonian Monte Carlo algorithm to sample from each marginal posterior density. Estimates are obtained with 1200 iterations, including a warm up of 500 iterations. The discretization time step $\Delta$ is equal to $5 \mathrm{~s}$.

\section{Computational validation of the approach}

In this section, we show the validation of the proposed approach on some simulated instances that replicate three different HD sessions, one for each HD technique (SHD, HDF-pre and HDF-post).

\section{I Simulated instances}

In each session, the values of the state variables $V_{p l}, V_{i s}, M_{e x}^{(s)}$ and $M_{i c}^{(s)}$ at each $t_{h}$ have been simulated with the discretized model, considering mean physiological values for the parameters (both patient-specific and nonpatient-specific parameters) and initial values for the state variables from a real session. 


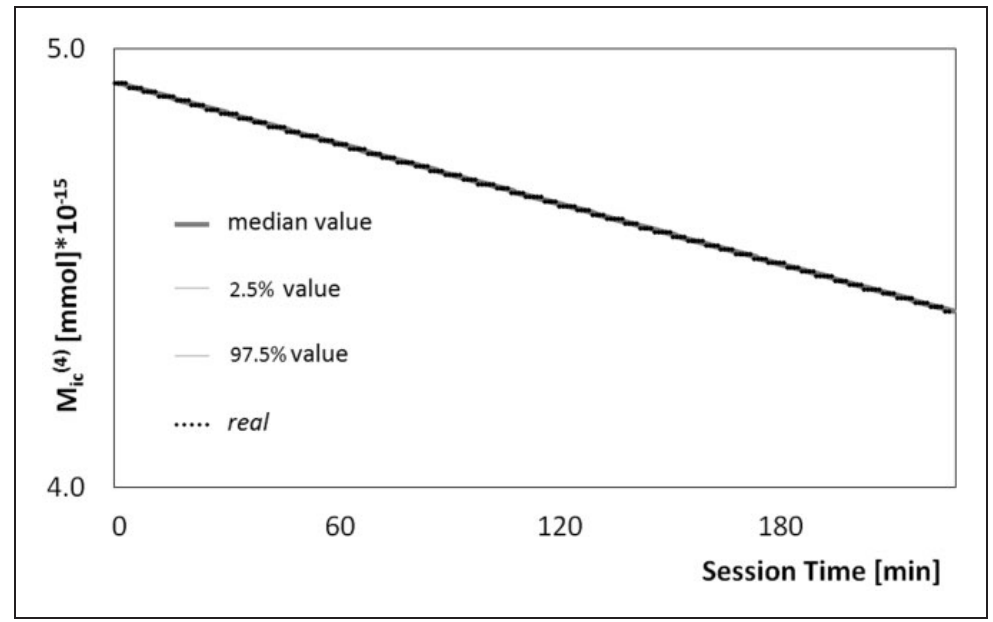

Figure 7. Comparison between simulated and estimated trends for intracellular mass of calcium $M_{i c}^{(4)}$ (c) for HDF-pre in the HFO case - cross check of parameter $k^{(4)}$.

The values adopted for the patient-specific parameters are reported in the last column of Tables 1-3. The initial values were acquired within the DialysIS Project at the Nephrology and Dialysis Unit of the A. Manzoni Hospital, (Lecco, Italy) for the SHD session, at the Nephrology and Dialysis Unit of the Regional Hospital of Lugano (Lugano, Switzerland) for the HDF-pre session, and at the Nephrology and Dialysis Unit of the Sant'Anna Hospital (Como, Italy) for the HDF-post session.

The integration of ODE system in equations (1) to (7) has been performed with the same 4th order Runge-Kutta method used for the estimation approach. The discretized system for simulating values has been implemented in Matlab (The MathWorks Inc., Natick, MA, USA), with a discretization step $\Delta$ of $5 \mathrm{~s}$ and a total duration of $227 \mathrm{~min}$ for HDF-pre, $240 \mathrm{~min}$ for SHD, and $264 \mathrm{~min}$ for HDF-post (equal to the length of the respective treatment).

\subsection{Validation approach}

The goal of the validation is to use the simulated values to estimate the patient-specific parameters. Under the same initial values of the state variables and the non-patient-specific parameters, we estimate the posterior densities for the patient-specific parameters with the proposed approach, and we compare them with their true values used to generate the simulated observations.

Two cases are considered for each simulated session, namely a high frequency observations (HFO) case and a low frequency observations (LFO) case.

For the HFO case, the interval between two consecutive observations $t_{h^{*}}-t_{h^{*}-1}$ is equal to 1 min for all variables. Thus, as $\Delta=5 \mathrm{~s}$, one value every 20 simulated values has been taken.

The LFO case reproduces the clinical conditions, where it is impossible to register all the variables with a high frequency (e.g. every minute). In particular, we replicate the worst clinical conditions in terms of acquisition frequency, namely:

- $V_{p l}(t)$ is taken every minute;

- $V_{i s}(t)$ is taken every hour;

- $M_{e x}^{(s)}(t)$ and $M_{i c}^{(s)}(t)$ are taken only at the beginning and the end of the treatment.

While HFO analyzes the approach in the presence of a high number of observations, as commonly in the literature, ${ }^{23,25,26}$ LFO considers a realistic clinical setting. The goal is to quantify possible detriments of the estimates under commonly adopted acquisition frequencies.

\subsection{Results}

We first consider the convergence of the MCMC chain and the computational times; then, we analyze the estimates to assess the goodness of the approach. 
Table 10. Marginal posterior densities of model parameters (SHD, real patient).

\begin{tabular}{llllll}
\hline Parameter & Min & $25 \%$ & $50 \%$ & $75 \%$ & Max \\
\hline$\eta^{(1)}-$ sodium & 0.00 & 0.01 & 0.02 & 0.04 & 0.20 \\
$\eta^{(2)}-$ potassium & 0.00 & 0.05 & 0.13 & 0.26 & 0.94 \\
$\eta^{(3)}-$ chloride & 0.00 & 0.01 & 0.03 & 0.76 & 0.29 \\
$\eta^{(4)}-$ calcium & 0.00 & 0.26 & 0.53 & 0.07 & 1.00 \\
$\eta^{(5)}-$ bicarbonate & 0.00 & 0.02 & 0.03 & 0.63 & 0.62 \\
$\eta^{(6)}-$ magnesium & 0.00 & 0.19 & 0.38 & 0.27 & 1.00 \\
$\eta^{(7)}-$ urea & 0.00 & 0.04 & 0.13 & 0.69 & 0.95 \\
$\eta^{(8)}-$ creatinine & 0.00 & 0.18 & 0.40 & 2.87 & 1.00 \\
$\rho$ & 2.46 & 2.71 & 3.79 & $3.59 \cdot 10^{-3}$ & 3.15 \\
$k^{(1)}-$ sodium & $2.52 \cdot 10^{-3}$ & $3.20 \cdot 10^{-3}$ & $1.96 \cdot 10^{-4}$ & $1.68 \cdot 10^{-4}$ & $4.45 \cdot 10^{-3}$ \\
$k^{(2)}-$ potassium & $1.45 \cdot 10^{-4}$ & $1.85 \cdot 10^{-4}$ & $1.56 \cdot 10^{-4}$ & $1.77 \cdot 10^{-4}$ & $2.61 \cdot 10^{-4}$ \\
$k^{(3)}-$ chloride & $1.18 \cdot 10^{-4}$ & $1.46 \cdot 10^{-4}$ & $1.65 \cdot 10^{-4}$ & $2.05 \cdot 10^{-4}$ \\
$k^{(4)}-$ calcium & $1.15 \cdot 10^{-4}$ & $1.54 \cdot 10^{-4}$ & $2.54 \cdot 10^{-3}$ & $1.78 \cdot 10^{-4}$ & $2.22 \cdot 10^{-4}$ \\
$k^{(5)}-$ bicarbonate & $1.87 \cdot 10^{-3}$ & $2.35 \cdot 10^{-3}$ & $1.67 \cdot 10^{-4}$ & $3.63 \cdot 10^{-3}$ \\
$k^{(6)}$ - magnesium & $1.21 \cdot 10^{-4}$ & $1.57 \cdot 10^{-4}$ & $3.93 \cdot 10^{-3}$ & $2.18 \cdot 10^{-4}$ \\
$k^{(7)}-$ urea & $3.30 \cdot 10^{-3}$ & $3.76 \cdot 10^{-3}$ & $1.42 \cdot 10^{-4}$ & $1.52 \cdot 10^{-4}$ & $2.87 \cdot 10^{-3}$ \\
$k^{(8)}-$ creatinine & $9.40 \cdot 10^{-5}$ & $1.32 \cdot 10^{-4}$ & $2.02 \cdot 10^{-4}$ \\
\hline
\end{tabular}

Table II. Marginal posterior densities of model parameters (HDF-post, real patient).

\begin{tabular}{|c|c|c|c|c|c|}
\hline Parameter & Min & $25 \%$ & $50 \%$ & $75 \%$ & $\operatorname{Max}$ \\
\hline$\eta^{(1)}-$ sodium & 0.73 & 0.94 & 0.97 & 0.99 & 1.00 \\
\hline$\eta^{(2)}-$ potassium & 0.05 & 0.82 & 0.91 & 0.96 & 1.00 \\
\hline$\eta^{(3)}-$ chloride & 0.00 & 0.24 & 0.35 & 0.47 & 0.91 \\
\hline$\eta^{(4)}-$ calcium & 0.01 & 0.39 & 0.55 & 0.72 & 0.99 \\
\hline$\eta^{(5)}-$ bicarbonate & 0.36 & 0.81 & 0.90 & 0.95 & 1.00 \\
\hline$\eta^{(6)}-$ magnesium & 0.01 & 0.46 & 0.68 & 0.84 & 1.00 \\
\hline$\eta^{(7)}-$ urea & 0.68 & 0.95 & 0.97 & 0.99 & 1.00 \\
\hline$\eta^{(8)}-$ creatinine & 0.00 & 0.39 & 0.67 & 0.87 & 1.00 \\
\hline$\rho$ & 1.59 & 1.78 & 1.84 & 1.90 & 2.14 \\
\hline$k^{(1)}-$ sodium & $2.55 \cdot 10^{-3}$ & $3.32 \cdot 10^{-3}$ & $3.53 \cdot 10^{-3}$ & $3.74 \cdot 10^{-3}$ & $4.69 \cdot 10^{-3}$ \\
\hline$k^{(2)}-$ potassium & $1.48 \cdot 10^{-4}$ & $1.98 \cdot 10^{-4}$ & $2.11 \cdot 10^{-4}$ & $2.23 \cdot 10^{-4}$ & $2.79 \cdot 10^{-4}$ \\
\hline$k^{(3)}-$ chloride & $1.22 \cdot 10^{-4}$ & $1.51 \cdot 10^{-4}$ & $1.62 \cdot 10^{-3}$ & $1.74 \cdot 10^{-4}$ & $2.29 \cdot 10^{-3}$ \\
\hline$k^{(4)}-$ calcium & $1.24 \cdot 10^{-4}$ & $1.53 \cdot 10^{-4}$ & $1.64 \cdot 10^{-4}$ & $1.77 \cdot 10^{-4}$ & $2.16 \cdot 10^{-4}$ \\
\hline$k^{(5)}-$ bicarbonate & $2.71 \cdot 10^{-3}$ & $3.26 \cdot 10^{-3}$ & $3.48 \cdot 10^{-3}$ & $3.72 \cdot 10^{-3}$ & $4.61 \cdot 10^{-3}$ \\
\hline$k^{(6)}-$ magnesium & $1.22 \cdot 10^{-4}$ & $1.63 \cdot 10^{-4}$ & $1.74 \cdot 10^{-4}$ & $1.86 \cdot 10^{-4}$ & $2.42 \cdot 10^{-4}$ \\
\hline$k^{(7)}-$ urea & $2.41 \cdot 10^{-3}$ & $2.93 \cdot 10^{-3}$ & $3.08 \cdot 10^{-3}$ & $3.22 \cdot 10^{-3}$ & $3.78 \cdot 10^{-3}$ \\
\hline$k^{(8)}-$ creatinine & $9.03 \cdot 10^{-5}$ & $1.29 \cdot 10^{-4}$ & $1.39 \cdot 10^{-4}$ & $1.46 \cdot 10^{-4}$ & $2.03 \cdot 10^{-4}$ \\
\hline
\end{tabular}

Nice traceplots and autocorrelations are obtained for each session, showing a satisfactory convergence of the MCMC chain. Moreover, values $\hat{R}$ of the Gelman-Rubin convergence statistics are always equal or higher than 1 for each estimated parameter in $\Theta^{*}$, confirming the convergence of the chain. The computational times have been equal to $14.5 \pm 4.4$ and $14.9 \pm 6.7 \mathrm{~h}$ for the HFO and LFO tests, respectively, on a server equipped with processor X86-64 AMD Opteron 6328 and 64GB of RAM. These times are largely lower than the common interval between two consecutive HD sessions, which is a fundamental requirement to apply the method in the clinical practice, where patient-specific parameters must be estimated with the data of a session and then used to optimize the kinetic response of the following session.

We start the analysis considering the marginal posterior densities of the patient-specific parameters for the HFO case, compared with the true values used to generate the simulated datasets. Results are reported in Tables 1-3 showing that in most of the cases, the posterior $25-75 \%$ credible intervals contain the true value and that the order of magnitude is almost always correctly estimated. 


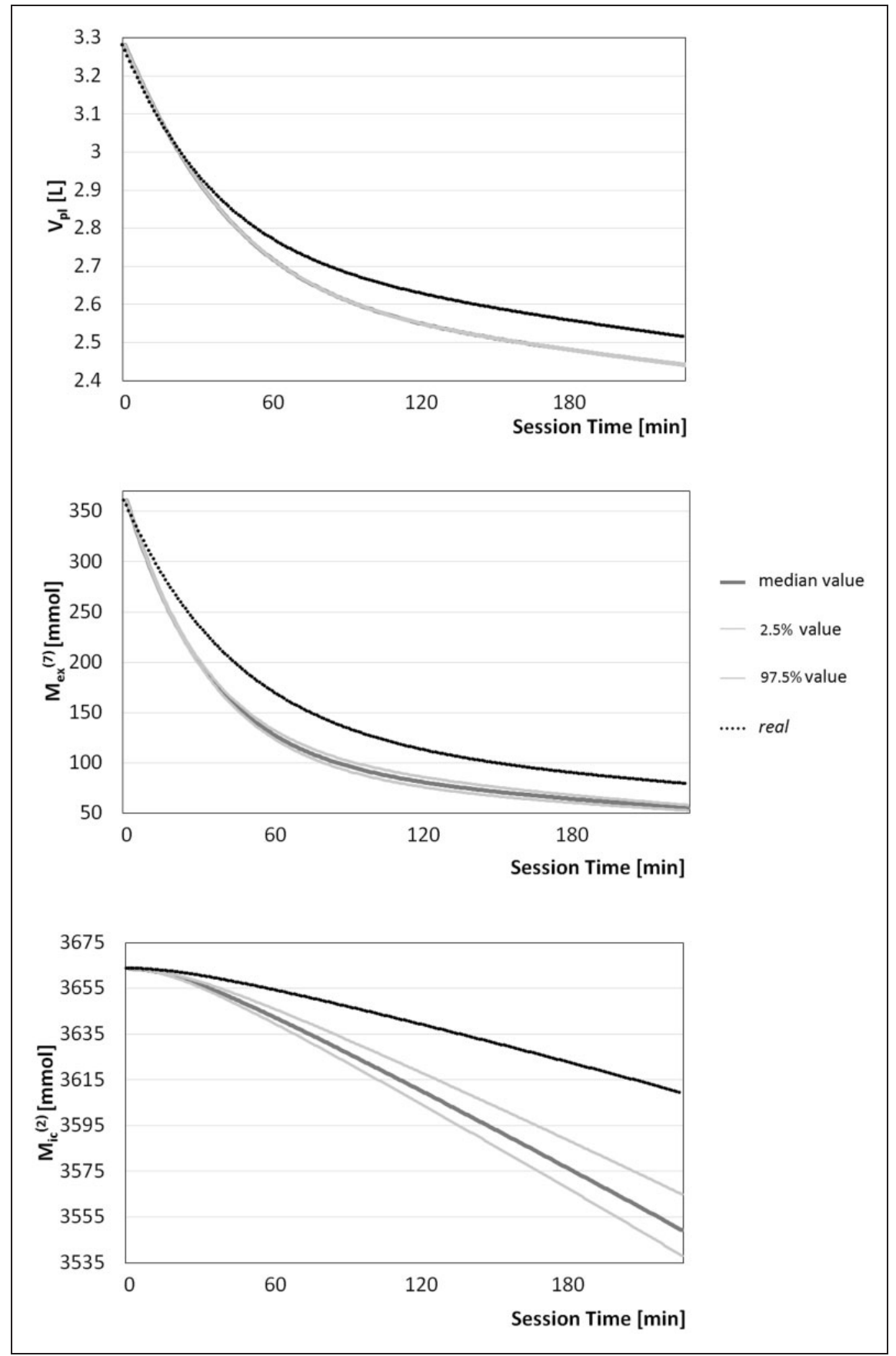

Figure 8. Comparison between simulated and estimated trends for plasmatic volume $V_{p l}(\mathrm{a})$, extracellular mass of urea $M_{\mathrm{ex}}^{(7)}(\mathrm{b})$, and intracellular mass of potassium $M_{i c}^{(2)}$ (c) for HDF-pre in the LFO case.

The only relevant difference concerns $k^{(4)}$ for calcium, for which our estimation is several orders of magnitude lower than the true value in all of the three cases. This means that the estimated model discharges the contribution of the calcium, which is nullified by the almost null coefficient $k^{(4)}$. This neglected contribution may either result in a bad or in an acceptable reconstruction of the temporal profiles of the state variables. In the first case, this would invalidate the approach; in the latter, the dynamics are not sensitive to the value of $k^{(4)}$, and this parameter is not identifiable from the observations.

To validate the approach, we compare the simulated (generated with the true values) and the estimated trends for three state variables, i.e. a volume, an extracellular mass and an intracellular mass. Results are reported in 


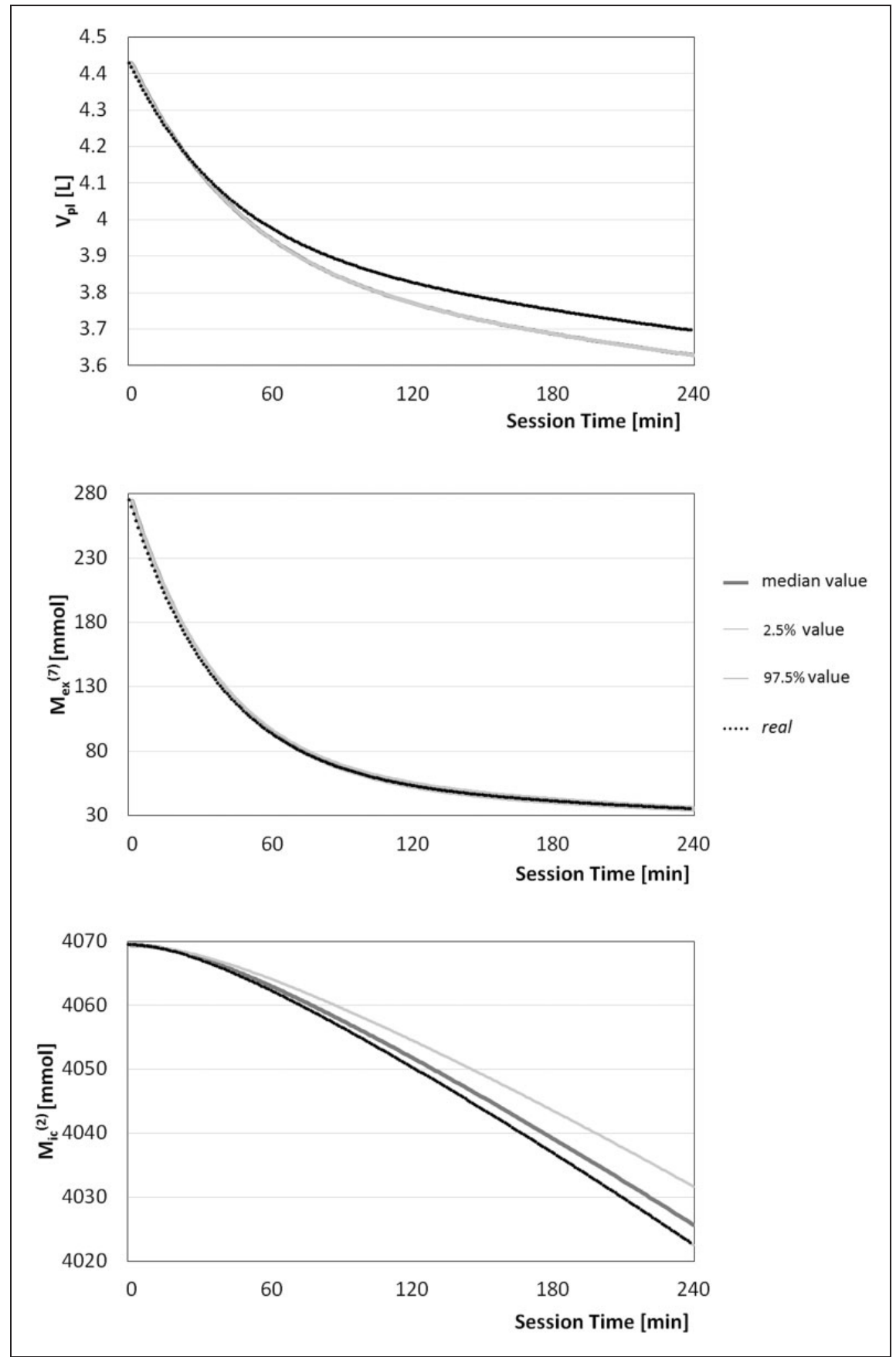

Figure 9. Comparison between simulated and estimated trends for plasmatic volume $V_{p l}(a)$, extracellular mass of urea $M_{\mathrm{ex}}^{(7)}(\mathrm{b})$, and intracellular mass of potassium $M_{i c}^{(2)}$ (c) for SHD in the LFO case.

Figures 2-4. As the estimated trends are stochastic, due to the posterior densities of the estimated parameters, they are reported in terms of their median value and 2.5-97.5\% credible band. Indeed, 100 samples are extracted from the joint posterior density of the patient-specific parameters, and the discretized model has been run as many times for each set of extracted values. Then, trends are combined for each discrete time instant, and the trends corresponding to the median value and the $2.5-97.5 \%$ credible band are taken. Results show a good reproducibility of the simulated datasets, with low differences between the simulated and the estimated trends. Very strict $2.5-97.5 \%$ credible bands are also obtained, proving the capability to reproduce the dynamics with a low degree of uncertainty. The same comparison is shown in Figure 5 for the calcium 


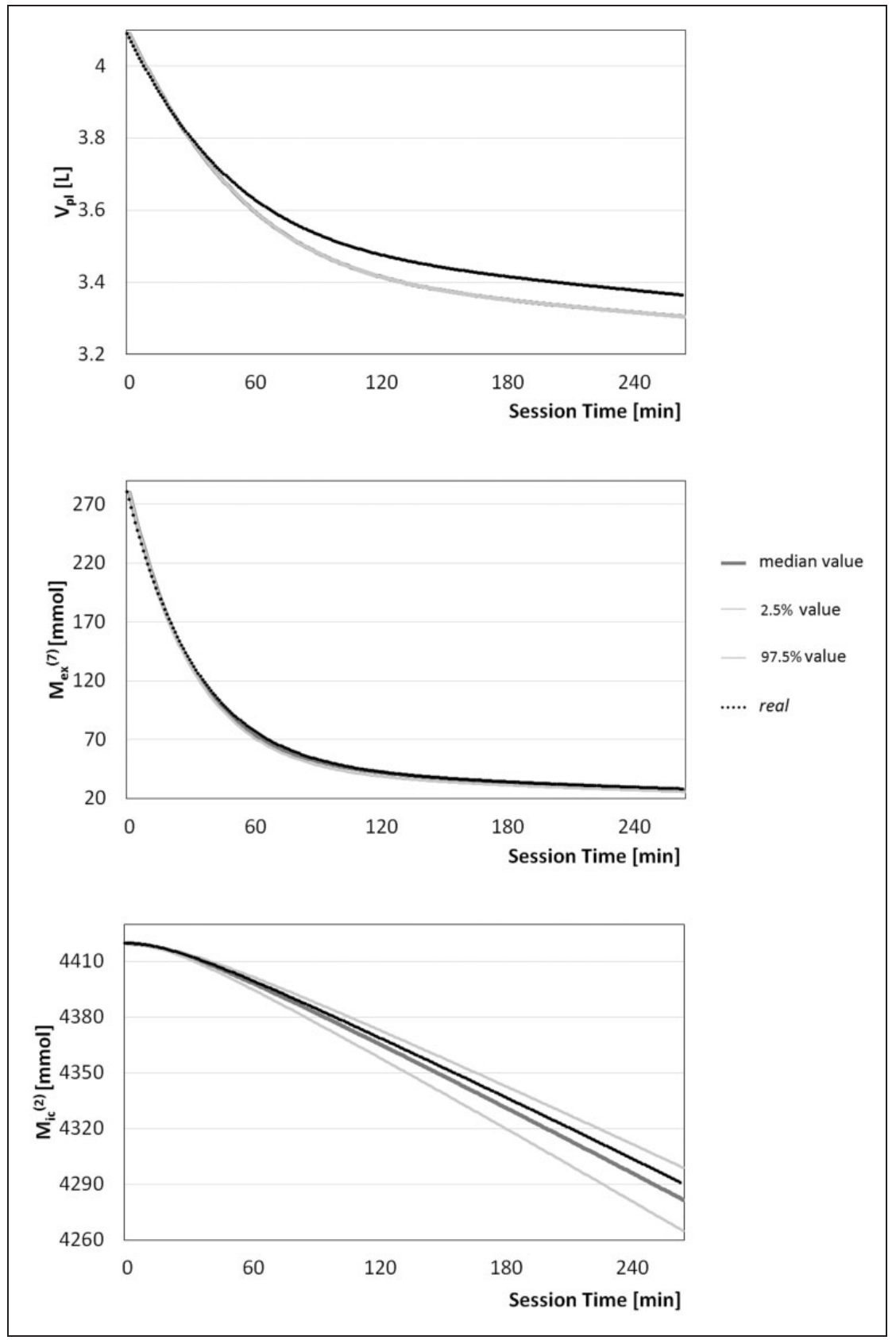

Figure 10. Comparison between simulated and estimated trends for plasmatic volume $V_{p l}(\mathrm{a})$, extracellular mass of urea $M_{\mathrm{ex}}^{(7)}(\mathrm{b})$, and intracellular mass of potassium $M_{i c}^{(2)}$ (c) for HDF-post in the LFO case.

intracellular mass $M_{i c}^{(4)}$. Results show that the estimation error of parameter $k^{(4)}$ marginally affects the estimated dynamics of this specific solute, with a difference between the simulated and the estimated value below the $10 \%$ at the end of the treatment.

Finally, we cross check the impact of the altered parameter $k^{(4)}$ by reconstructing the dynamics of the state variables with the true value of $k^{(4)}$ and the estimated values of the other parameters (extracted 100 times from the posterior density as for the analysis above), for the HDF-pre treatment. We obtain similar trends with comparable errors in all other trends (Figure 6 with respect to Figure 2), and a null error for the intracellular mass of calcium $M_{i c}^{(4)}$ (Figure 7 with respect to Figure 5). There results definitely confirm that, despite the lower order of magnitude estimated for $k^{(4)}$, this parameter does not affect the dynamics of the whole system besides calcium. 

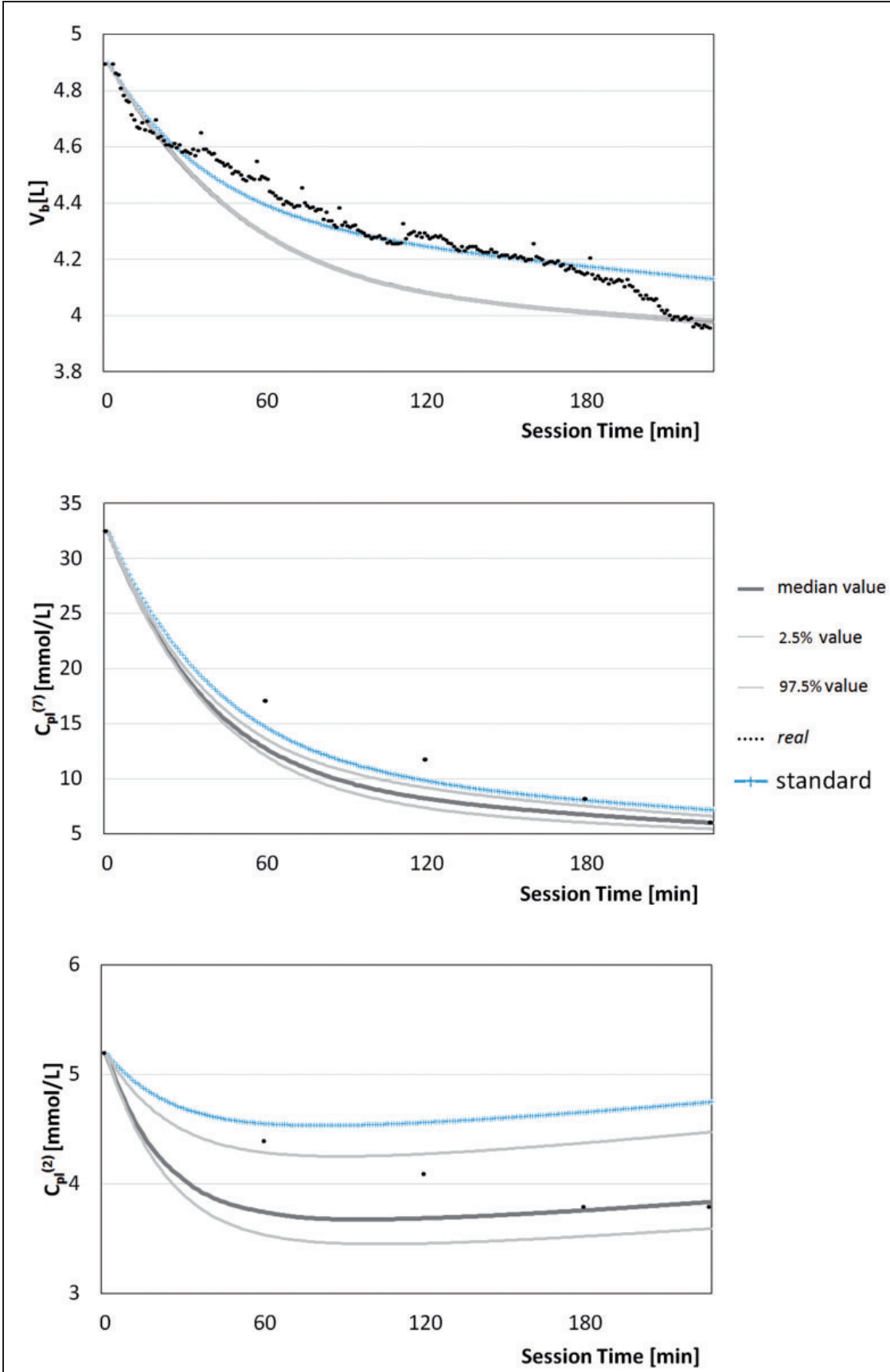

Figure II. Comparison between estimated trends and real clinical registrations for blood volume $V_{b}(a)$, plasma urea concentration $C_{p l}^{(7)}(b)$, and plasma potassium concentration $C_{p l}^{(2)}(c)$ for the HDF-pre treatment.

In addition, we remark that calcium plays a crucial role in the cardiovascular stability and that the knowledge of its compartmentalization, which is supposed to be different from that of the other electrolytes, is still limited. This fact is proved by the presence of kinetic models specific for calcium. ${ }^{16}$

We now consider the LFO case. The marginal posterior densities of the patient-specific parameters for these tests, compared with the true values used to generate the simulated datasets, are reported in Tables 4-6. Not only the estimates are not deteriorated by the lower amount of information, but it is possible to observe that the order of magnitude is always correctly estimated, also for $k^{(4)}$. Probably, in the presence of a lower amount of 


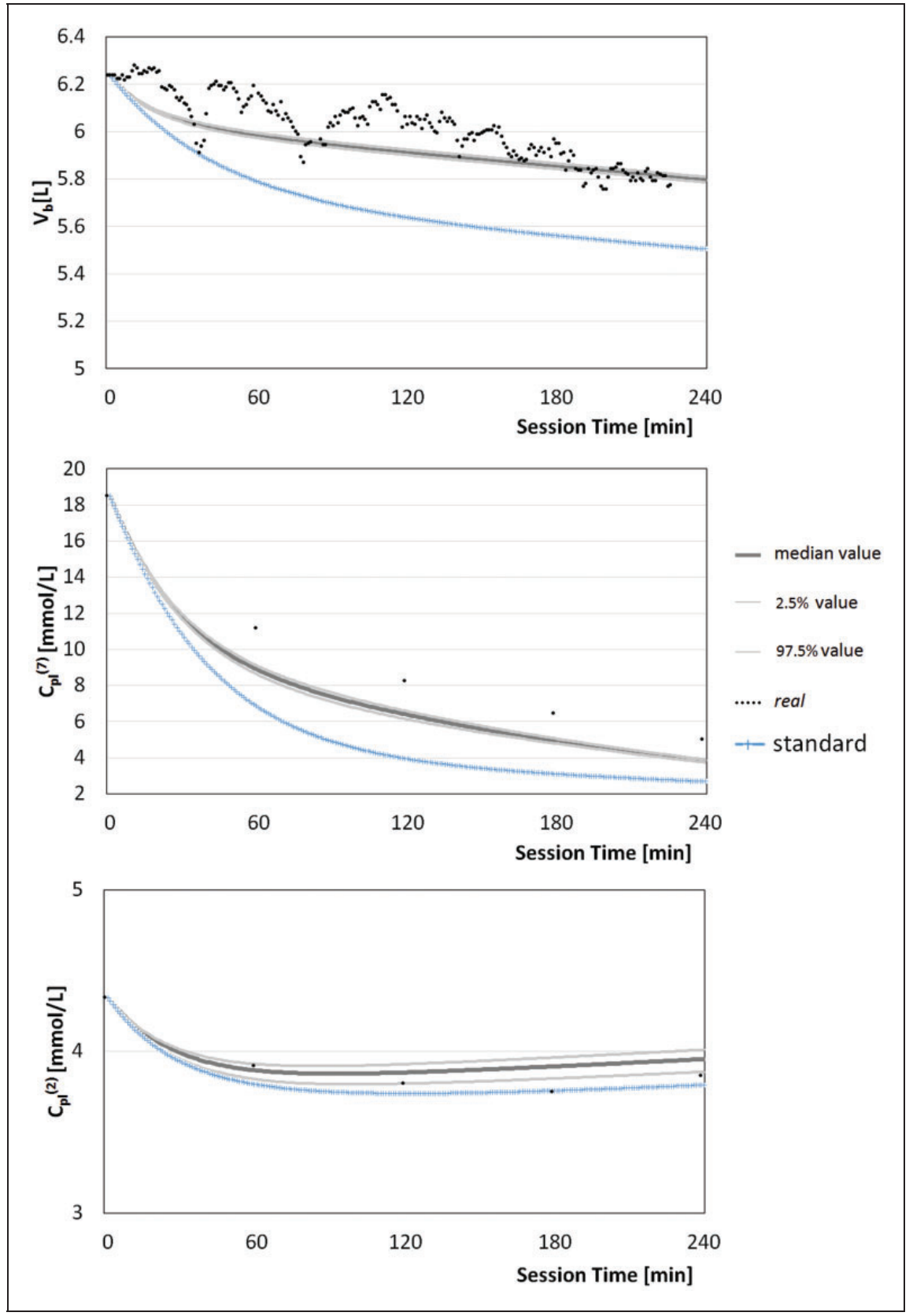

Figure 12. Comparison between estimated trends and real clinical registrations for blood volume $V_{b}(a)$, plasma urea concentration $C_{p l}^{(7)}(b)$, and plasma potassium concentration $C_{p l}^{(2)}(\mathrm{c})$ for the SHD treatment.

information, the contribution related to calcium plays a significant role in determining the response, and it is not discharged any more from the model.

The comparisons between the simulated and the estimated trends are reported in Figures 8-10. We can observe that the estimation errors are only slightly higher if compared with those obtained under the HFO case. This confirms that we may characterize a patient in realistic clinical conditions, when few clinical data are available.

\section{Application to real patients}

After validation, the proposed Bayesian approach has been applied to real patients to test the ability of providing fair estimations in line with patients' characteristics under real measurements. 

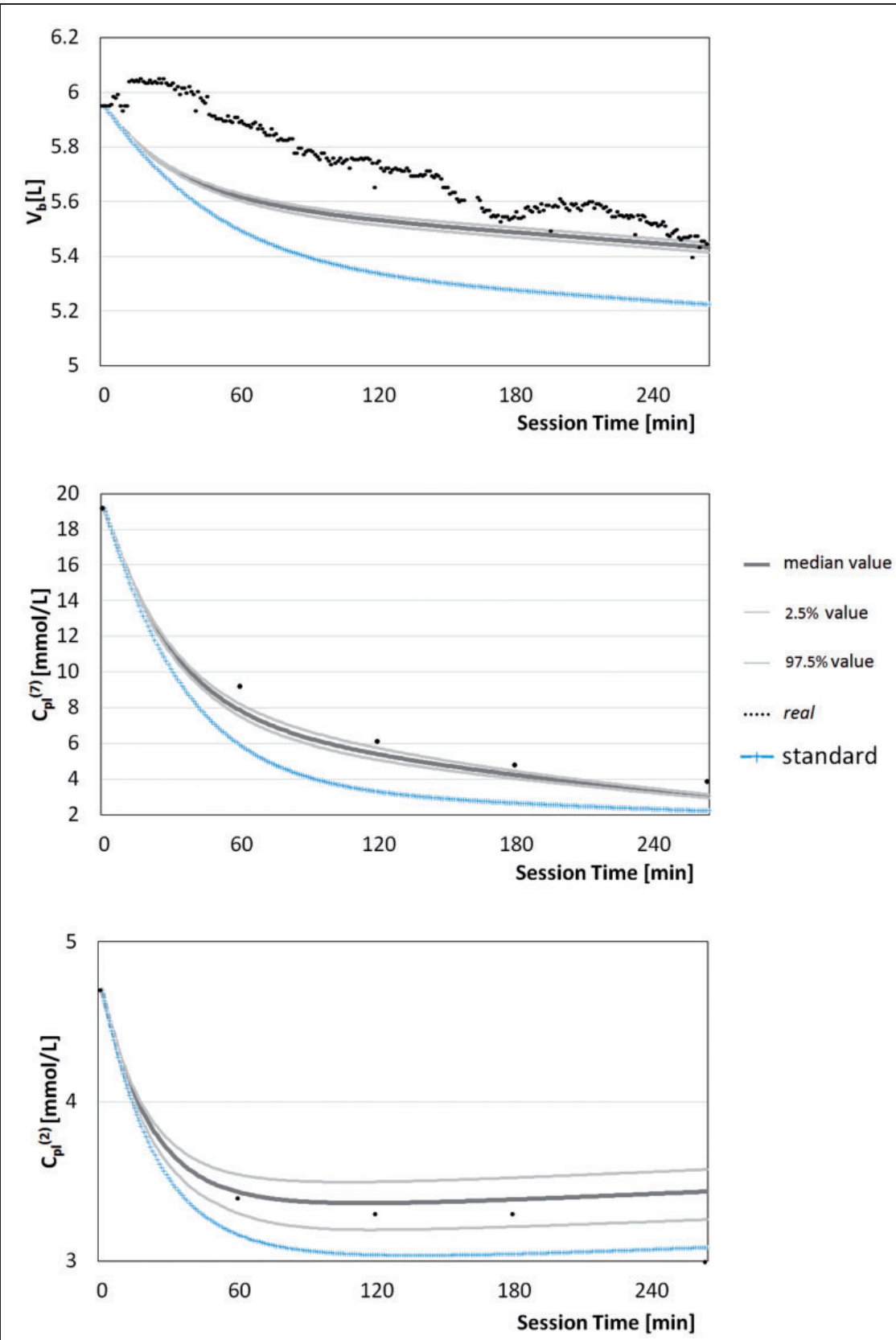

Figure 13. Comparison between estimated trends and real clinical registrations for blood volume $V_{b}(a)$, plasma urea concentration $C_{p l}^{(7)}(b)$, and plasma potassium concentration $C_{p l}^{(2)}$ (c) for the HDF-post treatment.

We consider three patients, one for each type of HD treatment. These patients are the same used to get the initial values of the state variables for the validation. Patients' characteristics are reported in Table 7.

As common in clinical studies, some time-dependent variables have been acquired during the treatment with different frequencies. Those acquired in this study are reported in Table 8 . Then, the observations of the state variables have been derived using the available clinical data, by means of the equations reported in the Supplementary Material; this additional step is made necessary because it is impossible to directly measure the state variables in clinics. Due to the acquisition frequencies of Table 8 , the available dataset in terms of state variables is as follows: 


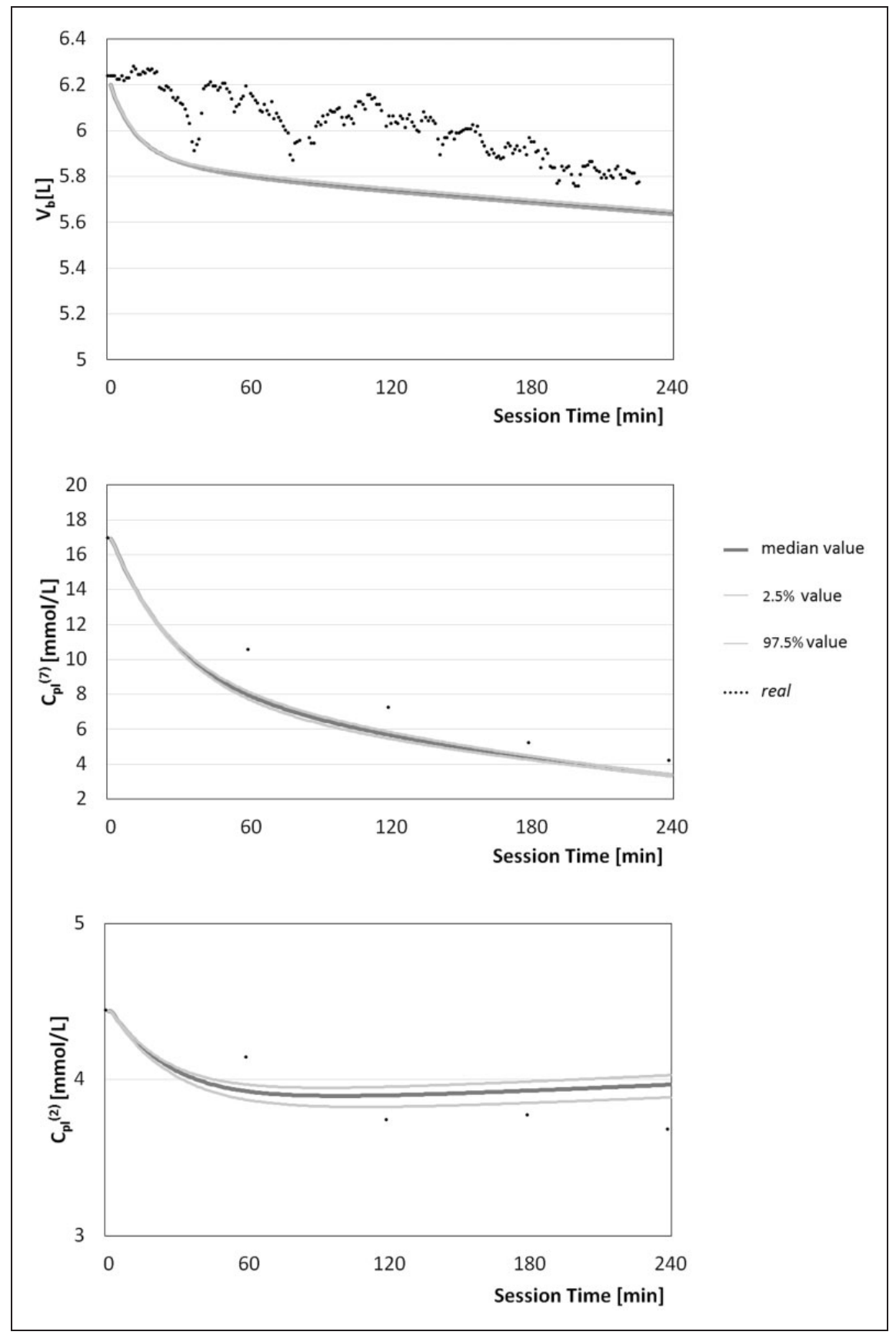

Figure 14. Comparison between estimated trends and real clinical registrations for blood volume $V_{b}(a)$, plasma urea concentration $C_{p l}^{(7)}(b)$, and plasma potassium concentration $C_{p l}^{(2)}(c)$ for the SHD treatment ( $2^{\text {nd }}$ monitored session).

- $V_{p l}(t)$ taken every minute;

- $V_{i s}(t)$ taken every minute;

- $M_{e x}^{(s)}(t)$ and $M_{i c}^{(s)}(t)$ taken at the beginning and the ending of the treatment $\forall s$.

Results from the Bayesian approaches are in terms of the estimated patient-specific parameters (posterior densities) and the trends of the state variables (stochastic trends). Thus, to compare the trends with the actual 

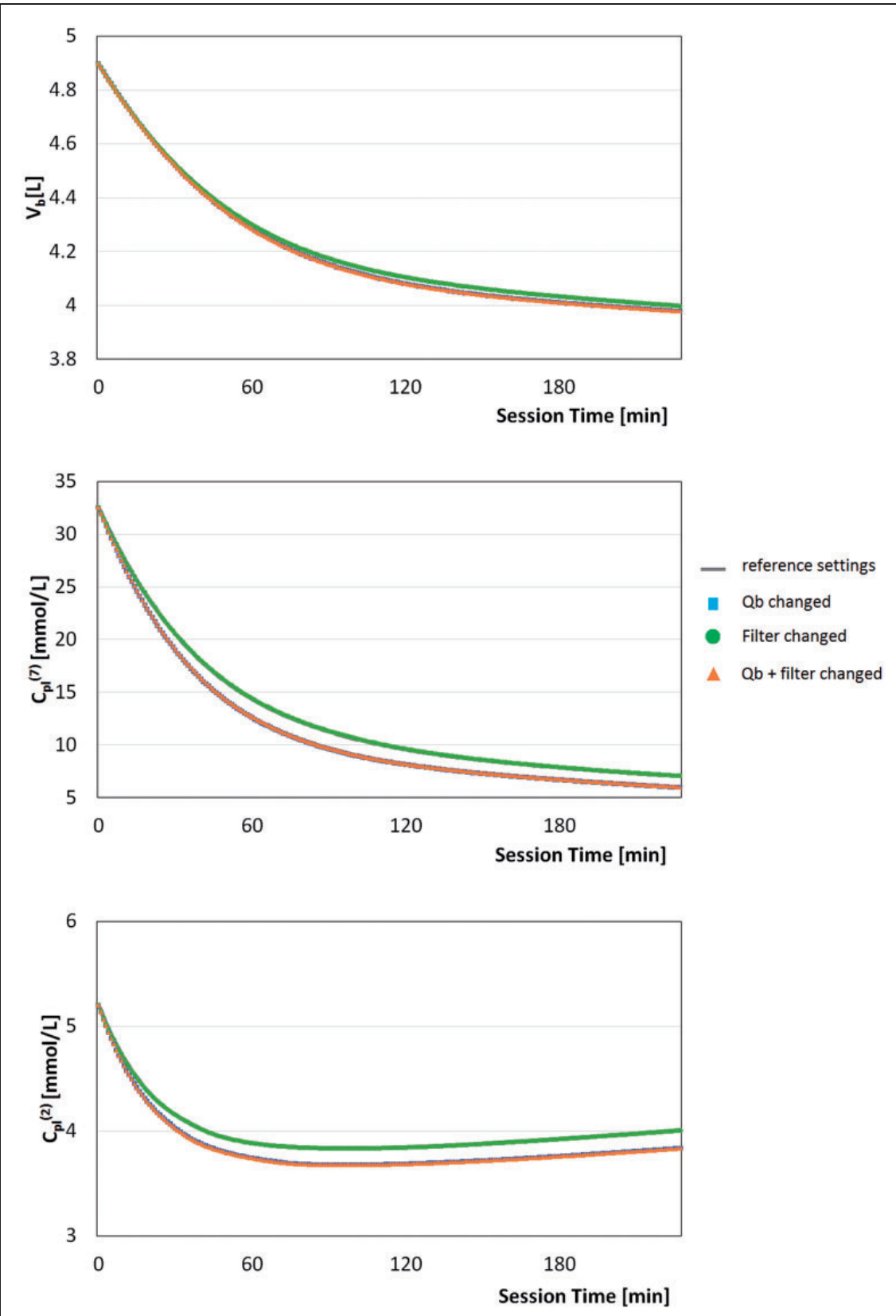

Figure 15. Comparison between estimated trends of blood volume, urea and potassium concentrations for the HDF-pre treatment and the same trends obtained changing some treatment settings.

clinical data, volumes and masses $\left(V_{p l}(t), V_{i s}(t), M_{e x}^{(s)}(t)\right.$ and $\left.M_{i c}^{(s)}(t)\right)$ are translated into plasmatic concentrations $\left(C_{p l}^{(s)}(t)\right)$ by means of the inverse equations of those reported in the Supplemental Material.

The simulated trends have been also compared with those obtained by using standard parameters, taken by literature. Indeed, these trends are obtained with the same model but using standard parameters (which are deterministic values). Standard parameters are the ones reported in the last column of Tables 1-3.

\section{I Results}

Nice traceplots and autocorrelations are obtained for each session, as for the validation, showing a satisfactory convergence of the MCMC chain. Once again, values $\hat{R}$ of the Gelman-Rubin convergence statistics are always 
equal or higher than 1 for each parameter in $\Theta^{*}$, confirming the convergence. Finally, using the same server of that of the validation, a computational time equal to $6.4 \pm 6.3 \mathrm{~h}$ is observed.

The marginal posterior densities of the patient-specific parameters are reported in Tables 9-11. Results show larger credible intervals than those obtained for the simulated datasets, as expected, but they are however strict enough to identify the values. Moreover, the estimated values are in line with the expectations and the literature data, assessing the goodness of fit.

To validate the estimates, we compare the estimated trends for three clinical variables (blood volume, plasma urea and potassium concentrations) with the related acquired data. Results are reported in Figures 11-13. As mentioned above, the trends are derived from the estimated values of the state variables according to the inverse equations of those reported in the Supplemental Material. The stochastic trends are generated as for the validation, extracting 100 samples from the joint posterior density of the patient-specific parameters and combining the 100 trends at each discrete time instant. In the figures, they are reported in terms of median value and $2.5-97.5 \%$ credible band.

Results show a good fitting, with low differences between estimated and clinical data. Moreover, very strict 2.5-97.5\% credible bands are obtained, showing the capability of reproducing the trends with a low degree of uncertainty and confirming the stability of the proposed approach. Results also show a better fitting of clinical data when compared with the trends obtained with standard parameter values, i.e. better estimated trends are found using the patient-specific parameters from the Bayesian approach.

\section{Discussion and conclusion}

In this paper, we present a Bayesian estimation approach for HD, to estimate the patient-specific parameters of a multi-compartment model, with the goal of improving HD treatment customization.

Results from the computational validation show good performance of the approach, i.e. the capability to detect the real values of the parameters and efficiently fit the trajectories, even when the acquisition frequency of the observed variables is low. Further results from the application to real patients demonstrate that the proposed approach is fair and provides good estimates.

The model can be a useful tool to support clinical decisions and prescription changes. In the following, we briefly show how to use the patient-specific version of the kinetic model as a clinical support tool, thanks to the identified patient-specific parameters. Two examples are reported.

The first evaluation deals with the possibility to predict the single patient kinetic response to the next treatment given the patient-specific parameters estimated in the previous one, by assuming that these parameters are quite stable from HD session to HD session. For example, the parameters estimated for the SHD patient (see Table 10) have been used to generate the kinetic response in a second session of the same patient, whose initial conditions are different. Results in Figure 14 show good performances in fitting the clinical data, thus confirming that the estimated parameters can be kept for the next session and that clinicians have an effective tool to predict the patient-specific kinetic response.

The second evaluation deals with the optimization of the HD treatment, once assumed that the patientspecific parameters remain stable between consecutive HD treatments. The presence of a patient-specific kinetic model allows to rapidly evaluate the impact of potential changes in the prescription before the treatment starts. For example, the impact of changing (i) the blood flow rate, (ii) the dialysis filter, and (iii) both these parameters is shown in Figure 15 for the real HDF-pre patient of the previous Section. Indeed, it was of clinical interest to change the blood flow rate $\left(Q_{b}\right)$ from $300 \frac{\mathrm{ml}}{\mathrm{min}}$ to $350 \frac{\mathrm{ml}}{\mathrm{min}}$ and the dialyzer from a FX100 Cordiax to FX80 Cordiax (lower clearances, Fresenius Medical Care AG and Co. KGaA, Germany), to check how the blood volume, the plasma urea and potassium concentrations trends change. Results show that, by changing the blood flow rate or the blood flow rate and the dialysis filter simultaneously, it is possible to obtain better profiles, associated with lower values of $V_{b}, C_{p l}^{(7)}$ and $C_{p l}^{(2)}$ at the end of the HD treatment. This means that, with the alternative treatment settings, we can get values of these variables that are closer to the clinical target. In particular, it is possible to notice that the reference settings curve and the filter changed curve are overlapped, as well as the $Q_{b}$ changed and $Q_{b}+$ filter changed curves.

These achievements will be even strengthened once the tests will be performed on a wider number of HD sessions.

To conclude, let us remark that the goal of the proposed approach is to find out patient-specific parameters to tailor the HD treatment on the single patient. For example, we may estimate the parameters from the first HD treatment to better design the second treatment and so on, updating the information if the patient's characteristics 
change. Anyway, the Bayesian approach can easily include observations from multiple patients or multiple HD treatments on the same patient, by simply including observations from different treatments in the likelihood function (equation (10)). In case of multiple HD treatments on the same patient, this can be useful to include a higher amount of data to estimate the patient-specific parameters (see, e.g., Lanzarone et al. ${ }^{28}$ for a multi-period application in another context).

\section{Acknowledgement}

The data acquisition protocol and the informed consent for all of the involved patients was approved by the ethics committees of A. Manzoni Hospital, (Lecco, Italy), the Regional Hospital of Lugano (Lugano, Switzerland) and the Sant'Anna Hospital (Como, Italy).

\section{Declaration of conflicting interests}

The author(s) declared no potential conflicts of interest with respect to the research, authorship, and/or publication of this article.

\section{Funding}

The author(s) disclosed receipt of the following financial support for the research, authorship, and/or publication of this article: This work was performed in the frame of the cross border cooperation program INTERREG IT-CH 2007-2013: DialysIS (Dialysis therapy between Italy and Switzerland) Project (Grant no. 33570710). (www.dialysis-project.eu). The authors acknowledge the support of student Valeria Secchi, Politecnico di Milano, for the implementation of the Runge-Kutta discretization.

\section{Supplementary material}

Supplementary material is available for this article online.

\section{References}

1. ERA-EDTA Registry: ERA-EDTA Registry Annual Report 2015. Academic Medical Center, Department of Medical Informatics, Amsterdam, the Netherlands, 2017.

2. United States Renal Data System. 2016 USRDS annual data report: Epidemiology of kidney disease in the United States. National Institutes of Health, National Institute of Diabetes and Digestive and Kidney Diseases, Bethesda, MD, 2016.

3. Prakash S, Reddan D, Heidenheim P, et al. Central, pheripheral, and other blood volume changes during hemodialysis. ASAIO J 2002; 48: 379-382.

4. Tapolyai MB, Faludi M, Fülöp T, et al. Which fluid space is affected by ultrafiltration during hemodiafiltration? Hemodial Int 2014; 18: 384-390.

5. Daugirdas JT. Dialysis hypotension: a hemodynamic analysis. Kidney Int 1991; 39: 233-246.

6. Santos SF and Peixoto AJ. Sodium balance in maintenance hemodialysis. Semin Dial 2010; 23: 549-555.

7. Clark WR, Leypoldt JK, Henderson LW, et al. Quantifying the effect of changes in the hemodialysis prescription on effective solute removal with a mathematical model. J Am Soc Nephrol 1999; 10: 601-609.

8. Leypoldt JK, Kablitz C, Gregory MC, et al. Prescribing hemodialysis using a weekly urea mass balance model. Blood Purif 1991; 9: 271-284.

9. Casagrande G, Bianchi C, Vito D, et al. Patient-specific modeling of multicompartmental fluid and mass exchange during dialysis. Int J Artif Organ 2016; 39: 220-227.

10. Schneditz D and Daugirdas JT. Compartment effects in hemodialysis. Semin Dial 2001; 14: 271277.

11. Gotch FA and Keen M. Kinetic modeling in hemodialysis. In: Nissenson AR and Fine RN (eds) Clinical dialysis, 4th ed. New York, NY: McGraw-Hill, 2005.

12. Sargent JA and Gotch FA. Mathematic modeling of dialysis therapy. Kidney Int 1980; 18: S2S10.

13. Sausse A, Man NK and Funck-Brentano JL. A mathematical approach to hemodialysis therapy. Proc Eur Soc Artif Intern Organs 1974; 1: 8184.

14. Di Filippo S, Corti M, Andrulli S, et al. Optimization of sodium removal in paired filtration dialysis by single pool sodium and conductivity kinetic models. Blood Purif 1997; 15: $34-44$.

15. Leypoldt JK. Kinetics of $\beta 2$-microglobulin and phosphate during hemodialysis: effects of treatment frequency and duration. Seminar Dialys 2005; 18: 401-408. 
16. Di Filippo S, Carfagna F, La Milia V, et al. Assessment of intradialysis calcium mass balance by single pool variablevolume calcium kinetic model. Hemodialysis Int 2017; DOI: 10.1111/hdi.12531.

17. Agar BU, Culleton BF, Fluck R, et al. Potassium kinetics during hemodialysis. Hemodialysis Int 2015; 19: $23-32$.

18. Leypoldt JK, Agar BU, Cheung AK, et al. A pseudo-one compartment model of phosphorus kinetics during hemodialysis: further supporting evidence artificial organs. 2017 (in press).

19. Ursino M, Coli L, Magosso E, et al. A mathematical model for the prediction of solute kinetics, osmolarity and fluid volume changes during hemodiafiltration with on-line regeneration of ultrafiltrate (HFR). Int J Artificial Organ 2006; 29: 1031-1041.

20. Oksendal B. Stochastic differential equations: an introduction with applications, 6th ed. Berlin: Springer, 2003.

21. Kloeden PE and Platen E. Numerical solution of stochastic differential equations. Berlin: Springer, 1992.

22. Coelho FC, Codeço CT and Gomes MGM. A Bayesian framework for parameter estimation in dynamical models. PloS One 2011; 6: e19616.

23. Lanzarone E and Ruggeri F. Inertance estimation in a lumped-parameter hydraulic simulator of human circulation. J Biomech Eng T ASME 2013; 135: 061012.

24. Gilioli G, Pasquali S and Ruggeri F. Nonlinear functional response parameter estimation in a stochastic predator-prey model. Math Biosci Eng 2012; 9: 75-96.

25. Lanzarone E, Pasquali S, Mussi V, et al. Bayesian estimation of thermal conductivity and temperature profile in a homogeneous mass. Numer Heat Tr B-Fund 2014; 66: 397-421.

26. Auricchio F, Conti M, Ferrara A, et al. A clinically-applicable stochastic approach for non-invasive estimation of aortic stiffness using computed tomography data. IEEE T Bio-Med Eng 2015; 62: 176-187.

27. Spence MA, Blackwell PG and Blanchard JL. Parameter uncertainty of a dynamic multispecies size spectrum model. Can J Fisheries Aquatic Sci 2016; 73: 589-597.

28. Lanzarone E, Pasquali Gilioli G and Marchesini E. A Bayesian estimation approach for the mortality in a stage-structured demographic model. J Math Biol 2017; 75: 759-779.

29. Andrulli S, Colzani S, Mascia F, et al. The role of blood volume reduction in the genesis of intradialytic hypotension. Am J Kidney Dis 2002; 40: 1244-1254.

30. Ishibe $\mathrm{S}$ and Peixoto A. Methods of assessment of volume status and intercompartmental fluid shifts in hemodialysis patients: implications in clinical practice. Semin Dial 2004; 17: 37-43.

31. Sharma MK, Wieringa FP, Frijns AJH, et al. On-line monitoring of electrolytes in hemodialysis: on the road towards individualizing treatment. Expert Rev Med Devices 2016; 13: 933-943.

32. Colton CK, Henderson LW, Ford CA, et al. Kinetics of hemodiafiltration. I. In vitro transport characteristics of a hollowfiber blood ultrafilter. J Lab Clin Med 1975; 85: 355-371.

33. Ursino M, Colí L, Brighenti C, et al. Prediction of solute kinetics, acid-base status, and blood volume changes during profiled hemodialysis. Ann Biomed Eng 2000; 28: 204-216.

34. Stan Development Team: Stan modeling language users guide and reference manual, version 2.9.0. 2015.

35. Guyton AC and Hall JE. Textbook of medical physiology. Philadelphia, PA: Saunders, 2000.

\section{Appendix \\ Notation}

$C_{c} \quad$ capillary compliance $(\mathrm{L} / \mathrm{mmHg})$

$C_{i c}^{(s)}(t) \quad$ concentration of solute $s$ in the intracellular compartment $(\mathrm{mmol} / \mathrm{L})$

$C_{i n}^{(s)}(t) \quad$ concentration of solute $s$ in plasma water at the dialyzer inlet $(\mathrm{mmol} / \mathrm{L})$

$C_{i s}^{(s)}(t) \quad$ concentration of solute $s$ in the interstitial compartment $(\mathrm{mmol} / \mathrm{L})$

$E_{\text {is }} \quad$ interstitial elastance $(\mathrm{mmHg} / \mathrm{L})$

$H t(t)$ hematocrit (\%)

$k^{(s)}$ modulation of mass transfer efficiency across cell membrane for solute $s(\mathrm{~L} / \mathrm{s})$

$L_{c} \quad$ capillary permeability $(\mathrm{L} / \mathrm{mmHg} * \mathrm{sec})$

$M_{e x}^{(s)}(t) \quad$ extracellular mass of solute $s(\mathrm{mmol})$

$M_{i c}^{(s)}(t) \quad$ intracellular mass of solute $s(\mathrm{mmol})$

$P_{a c}(t)$ arterial capillary pressure $(\mathrm{mmHg})$

$P_{i s}(t) \quad$ interstitial pressure $(\mathrm{mmHg})$

$P_{n}(t) \quad$ Total net filtration pressure (difference between hydraulic and osmotic pressures) $(\mathrm{mmHg})$

$Q_{b}$ prescribed blood flow rate of the dialysis machine $(\mathrm{L} / \mathrm{s})$

$Q_{d i l}$ dilution flow rate present in HDF therapy (L/s) 
$Q_{f}$ filtration flow rate of the HD machine (L/s)

$Q_{\text {fcap }}(t) \quad$ Fluid flow rate across the capillary membrane $(\mathrm{L} / \mathrm{sec})$

$Q_{i c}(t) \quad$ Fluid flow rate across the cell membrane $(\mathrm{L} / \mathrm{s})$

$Q_{u f} \quad$ ultrafiltration flow rate across the dialyzer membrane $(\mathrm{L} / \mathrm{s})$

$s$ solute index: sodium $\mathrm{Na}^{+}(s=1)$; potassium $\mathrm{K}^{+}(s=2)$; chloride $\left(\mathrm{Cl}^{-} s=3\right)$; calcium $\mathrm{Ca}^{2+}(s=4)$; bicarbonate $\mathrm{HCO}_{3}^{-}(s=5)$; magnesium $\mathrm{Mg}^{2+}(s=6)$; urea $\mathrm{CO}\left(\mathrm{NH}_{2}\right)_{2} \quad(s=7)$; creatinine $\mathrm{C}_{4} \mathrm{H}_{7} \mathrm{~N}_{3} \mathrm{O}$ $(s=8)$

$t$ time index (s)

$T_{p}(t) \quad$ Total protein concentration in blood $(\mathrm{g} / \mathrm{dL})$

$V_{i c}(t)$ intracellular volume $(\mathrm{L})$

$V_{i s}(t)$ interstitial volume (L)

$V_{p l}(t)$ plasmatic volume (L)

$\beta \quad$ Ratio between intracellular and interstitial equilibrium concentrations ${ }^{35}$

$\eta^{(s)} \quad$ performance of dialyzer membrane in terms of filtration for solute $s$ [adimensional]

$\phi_{\text {dial }}^{(s)}(t) \quad$ molar flux of solute $s$ across the dialyzer ( $\mathrm{mmol} / \mathrm{s}$ )

$\phi_{\text {diff }}^{(s)}(t) \quad$ diffusive molar flux of solute $s$ across the dialyzer membrane $(\mathrm{mmol} / \mathrm{s})$

$\phi_{d i l}^{(s)} \quad$ molar dilution flux of solute $s(\mathrm{mmol} / \mathrm{s})$

$\phi_{i c}^{(s)}(t) \quad$ molar flux of solute $s$ across the cell membrane $(\mathrm{mmol} / \mathrm{s})$

$\rho$ modulation of fluid transfer efficiency across capillary membrane (adimensional) 